\title{
Boundary conditions and stability of a perfectly matched layer for the elastic wave equation in first order form
}

\author{
Kenneth Durua,d, Jeremy E. Kozdon ${ }^{\mathrm{b}}$, Gunilla Kreiss ${ }^{\mathrm{c}}$ \\ ${ }^{a}$ Department of Geophysics, Stanford University, Stanford, CA. \\ ${ }^{b}$ Department of Applied Mathematics, Naval Postgraduate School, Monterey, CA. \\ ${ }^{c}$ Division of Scientific Computing, Department of Information Technology, Uppsala University, Sweden. \\ ${ }^{d}$ Corresponding author: kduru@stanford.edu
}

\begin{abstract}
In computations, it is now common to surround artificial boundaries of a computational domain with a perfectly matched layer (PML) of finite thickness in order to prevent artificially reflected waves from contaminating a numerical simulation. Unfortunately, the PML does not give us an indication about appropriate boundary conditions needed to close the edges of the PML, or how those boundary conditions should be enforced in a numerical setting. Terminating the PML with an inappropriate boundary condition or an unstable numerical boundary procedure can lead to exponential growth in the PML which will eventually destroy the accuracy of a numerical simulation everywhere. In this paper, we analyze the stability and the well-posedness of boundary conditions terminating the PML for the elastic wave equation in first order form. First, we consider a vertical modal PML truncating a two space dimensional computational domain in the horizontal direction. We freeze all coefficients and consider a left half-plane problem with linear boundary conditions terminating the PML. The normal mode analysis is used to study the stability and wellposedness of the resulting initial boundary value problem (IBVP). The result is that any linear well-posed boundary condition yielding an energy estimate for the elastic wave equation, without the PML, will also lead to a well-posed IBVP for the PML. Second, we extend the analysis to the PML corner region where both a horizontal and vertical PML are simultaneous active. The challenge lies in constructing accurate and stable numerical approximations for the PML and the boundary conditions. Third, we develop a high order accurate finite difference approximation of the PML subject to the boundary conditions. To enable accurate and stable numerical boundary treatments for the PML we construct continuous energy estimates in the Laplace space for a one space dimensional problem and two space dimensional PML corner problem. We use summation-by-parts finite difference operators to approximate the spatial derivatives and impose boundary conditions weakly using penalties. In order to ensure numerical stability of the discrete PML, it is necessary to extend the numerical boundary procedure to the auxiliary differential equations. This is crucial for deriving discrete energy estimates analogous to the continuous energy estimates. Numerical experiments are presented corroborating the theoretical results. Moreover, in order to ensure longtime numerical stability, the boundary condition closing the PML, or its corresponding discrete implementation, must be dissipative. Furthermore, the numerical experiments demonstrate the stable and robust treatment of PML corners.
\end{abstract}

Keywords: elastic wave equation, first order systems, Rayleigh surface waves, perfectly matched layers, stability, normal mode analysis, high order finite difference, summation-by-parts, penalty method.

\section{Introduction}

Wave propagation problems are often formulated in unbounded or very large spatial domains. In numerical simulations, large spatial domains must be replaced by smaller computational domains by introducing artificial boundaries. Efficient and reliable domain truncation becomes essential, since it enables more accurate numerical simulations. More than thirty years of extensive research in this area has resulted in two standard, competing approaches for artificial boundary closures: high order local non-reflecting boundary condition (NRBC) [21]-[22], and damping layers such as the perfectly matched layer (PML) [1]-[12], grid stretching techniques [14] and others [13, 15]. An NRBC is a boundary condition defined on an artificial boundary such that little or no spurious reflections occur as a wave passes the boundary. In the methods based on damping layers the domain is extended to include a layer where waves are damped by some modification or transformation of the system. In this paper we will focus on PMLs, where the underlying equations are transformed such that waves traveling into the layer are absorbed without reflections. A very important property of the PML is perfect matching. This means that there are no reflections as waves propagate from the physical domain into the layer. It is also important to note that the perfect matching property of the PML is only guaranteed for the continuous model. When numerical approximations are introduced, the discrete PML can allow 
some numerical reflections. However, for a well designed numerical approximation, these artificial reflections vanish as the mesh size approaches zero.

Often, PMLs (and NRBCs) are derived by assuming a homogeneous unbounded medium. When the PML is used in semi-bounded domains, the PML introduces layer edges where the physical boundary conditions and the PML interact. This situation leads to an initial boundary value problem (IBVP) for the PML. A typical set-up can be found in $[6,10]$; where for the elastic wave equation a traction free boundary condition is used on one boundary with PML truncation on all others. Further complicating the matter, the PML is derived in the continuous setting under the assumption that it is an unbounded layer surrounding the truncated computational domain. However, in practice the PML is a layer of finite thickness. Thus, even when there is no physical boundary, the PML must be implemented as an IBVP. An effective PML boundary closure becomes important since it enables efficient numerical computations [11].

To be useful, any method for truncating a computational domain must not only be accurate but also inherit stability properties of the unbounded problem. In particular, if the original governing equation and its numerical approximations do not support growth, neither should the augmented system nor a corresponding numerical approximation. For hyperbolic systems, the temporal stability of the PML Cauchy problem is well known; see for example [3, 2]. When the domain is bounded or semi-bounded more care is required, since a PML which is stable in an unbounded domain, can support growth when boundaries are introduced [10,6]. Having said that, in [4] it was shown that the PML for second order elastic wave equations on a half plane with the free-surface boundary condition at a boundary tangential to the damping direction does not support temporally growing modes. A key step taken in [4] was to transform the derivatives in the free surface boundary condition using the PML metric. Subsequently, using the normal mode analysis, the PML with the transformed boundary condition was proven stable. However, the analysis in [4] considers only a physical boundary entering into the layer and the boundary condition terminating the PML at a boundary normal to the damping direction was not taken into consideration.

In this paper we work with the first order form of the equations, and the story is different. The application of the PML adds, in a nontrivial way, lower order (undifferentiated) terms to the elastic wave equation. Standard boundary conditions for first order hyperbolic systems contain a linear combination of the unknown variables, while boundary conditions for second order systems often involve combinations of the unknown variables and their spatial and temporal derivatives. In the second order case such boundary conditions are transformed in the PML, while in the corresponding first order case the transformation leaves the boundary conditions unaltered. Though one may naturally expect that a stable scheme for the interior IBVP can be extended to PML by simply adding the PML terms and augmenting the auxiliary equations without altering the numerical boundary procedure. However, numerical experiments reported in the literature demonstrate that growth may occur. In particular, numerical experiments in [6], using a staggered grid approximation, suggest that growth can occur for the split-field PML of [2] when a free-surface boundary condition is imposed. Stable numerical approximations of the PML are also a challenge to finite/spectral element methods $[8,15]$.

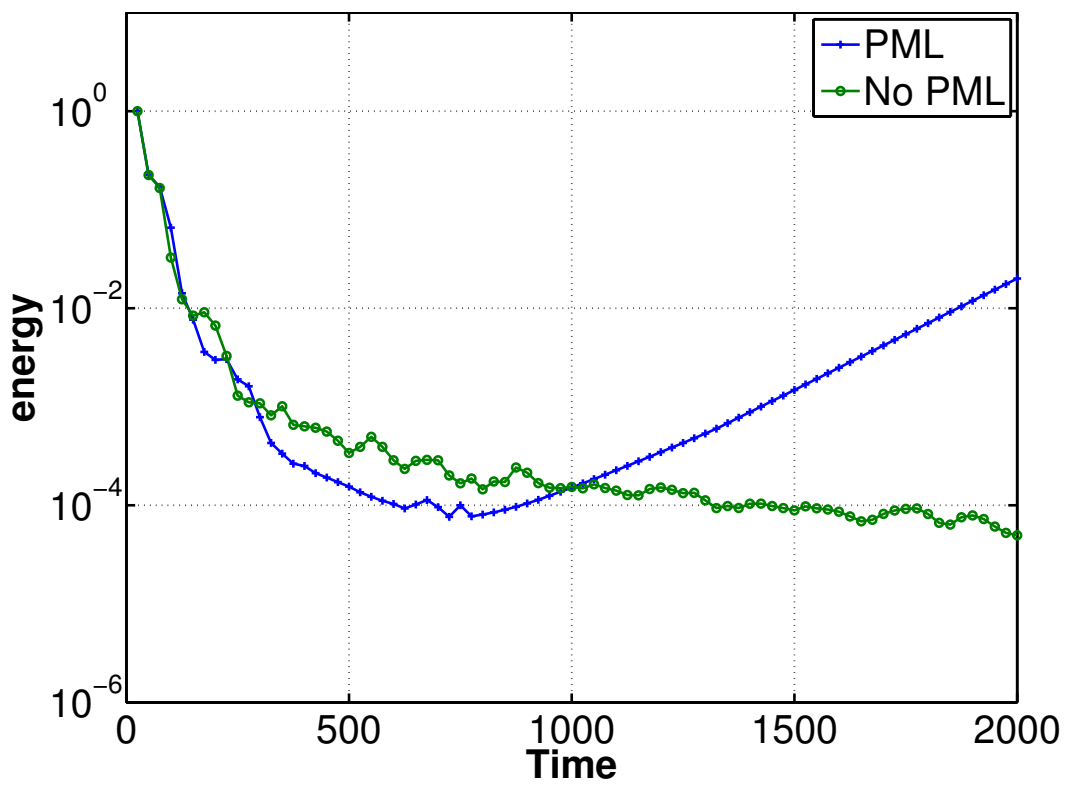

Figure 1: Time history of the maximum norm energy on the grid for aluminum waveguide with $c_{s} / c_{p}=0.4593$.

As an initial computation we consider a modal PML [3], with a damping profile that varies cubically, which 
is terminated by setting the incoming characteristics (corresponding to the one-dimensional problem in the normal direction) on the PML boundary to zero. We repeated the experiments of [6] for a two-dimensional aluminum solid with the free surface boundary conditions on the top surface of the solid, and PMLs along the other boundaries. The equations are written in first order form and are discretized using a high order finite difference scheme [20, 27], which is provably stable without the PML. No extra or different boundary conditions are imposed in the PML. For comparison we also compute without the PML. The time history of the discrete energy, resulting from our numerical experiments, is plotted in figure 1. Without the PML the energy decays throughout the computation, while for the PML case, the discrete energy decays until $t \sim 800$ and grows exponentially for $t>800$. Numerical instabilities similar to figure 1 was seen in [6] when the PML was used to truncate a half-plane elastic media with a free-surface condition at the boundary. Though both the experiments shown in figure 1 and [6] show instability, it is not obvious what causes the instability. In particular, is it inherent in the PML formulation on bounded domains, or a result of the numerical approximation?

A first step in answering this question is to consider the problem from a theoretical point of view. We freeze the coefficients of a two space dimensional (2D) modal PML [3] with damping in the horizontal direction, and consider separately the PML Cauchy problem, the lower half plane PML problem with the free surface boundary conditions at the upper horizontal boundary, and the left half plane PML problem corresponding to the vertical right outer edge of the PML. The Cauchy problem is known to be stable [3, 2], and will not be discussed further.

In the lower half plane problem, the free surface boundary interacts with the PML. In the first order case the PML metric transform leaves this boundary condition in the same form as without the PML. Stability of this lower half plane problem can be analyzed using normal mode analysis, and it is not surprising that this approach leads to the same analysis as for the second order formulation, see [4]. A first result is therefore that no growing modes are supported in the PML by the free surface boundary condition at a boundary parallel to the PML damping direction. In particular the analysis in [4] shows that Rayleigh waves modes are damped as they propagate along the boundary in the PML's damping direction.

The second result concerns the left half plane problem, for which investigate the stability of linear boundary conditions using normal mode analysis. The result is that any linear boundary condition, which guarantees bounded solution for the elastic wave equation without the PML, can be used without supporting growing modes in the PML. In particular, commonly used boundary conditions such as: Dirichlet, traction free and characteristic boundary conditions are fine. However, while the free surface boundary conditions support Rayleigh wave modes propagating in the tangential directions without decay, the characteristic boundary conditions ensure that all boundary wave modes are dissipated.

An important objective of this paper is to develop a systematic procedure to effectively enforce these boundary conditions in a PML in a discrete setting. The numerical method used in figure 1 is a high-order, summation-by-parts (SBP) finite difference scheme $[28,26,20,27]$. In these methods $[20,27]$, boundary conditions are enforced weakly using a penalty technique known as the simultaneous approximation term (SAT) method [23]. The power of the SBP-SAT method is that if the continuous problem satisfies an energy estimate, stability can be guaranteed by a careful choice of penalty parameters and establishing analogous discrete energy estimates. It can also be shown that discontinuous finite/spectral elements and nodal finite volume methods methods satisfy the SBP property $[18,29]$ and therefore can be interpreted as SBP-SAT schemes. Similar to SBP-SAT schemes, finite/spectral elements and nodal finite volume methods yield discrete energy estimates analogous to the continuous energy estimates of the partial differential equation (PDE).

The PML is a non-standard hyperbolic system, and for general systems like the elastic wave equation, there is no energy estimate for the PML, and hence no procedure of determining penalty parameters. In the computations presented in figure 1 we simply used the same penalties as without PML, where they yield a stable approximation. This approach can be disastrous as shown by figure 1. Clearly, good stability properties for the numerical method without the PML do not carry over once a PML is added, even if the PML is stable in the continuous setting.

Finally, we consider a one-dimensional model PML problem, and present a procedure to use this one dimensional model to derive SAT terms to be used in a two dimensional SBP finite difference scheme. The one-dimensional model includes only variations normal to the boundary, and it satisfies an energy estimate in the Laplace space. To ensure numerical stability of the discrete PML in a corresponding sense, it is crucial to be able to discretely mimic the derivation of the continuous energy estimate. The result is that, by including specific PML stabilizing penalties in the auxiliary equations we are able to derive discrete energy estimates that analogous to continuous energy estimates. A classification of dissipative and non-dissipative boundary procedures is also possible from this analysis.

The penalty terms can be directly applied in higher space dimensional settings, and two space dimensional numerical experiments verify that the PML stabilizing penalties are necessary also in two space dimensional settings. However, the two space dimensional experiments show that including the PML stabilizing penalties is sufficient to ensure long time stability. The final result, which we demonstrate numerically, is that the boundary condition (or its corresponding 
discrete implementation) must be dissipative [33]. The numerical experiments also demonstrate the stable and robust treatments of PML corners. Though the focus here is on SBP-SAT schemes, but the ideas developed in this paper can be extended to finite/spectral elements and nodal finite volume methods.

The remainder of the paper will proceed as follows. In section 2 we introduce the equations of linear elasticity and the corresponding PML equations. Stability of the continuous PML is analyzed in section 3. In section 4 we present numerical approximations and demonstrate numerical stability. Numerical experiments are presented in section 5 verifying the analysis of previous sections. We offer a brief conclusion in section 6 .

\section{Equations}

In this section, the equations of linear elasticity and the corresponding modal PML equations are introduced.

\subsection{Elastic wave equation}

Consider the two space dimensional equations of linear elastodynamics

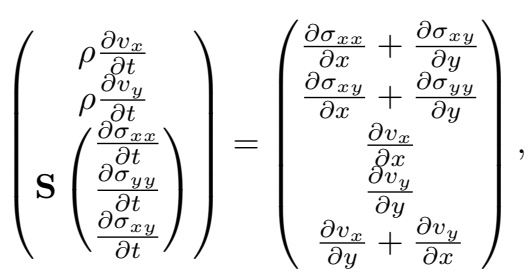

in an isotropic homogeneous elastic rectangular block, $-x_{0} \leq x \leq x_{0},-y_{0} \leq y \leq y_{0}$, with $x_{0}, y_{0}>0$, where the unknown $\mathbf{v}=\left(v_{x}, v_{y}\right)^{T}$ is the particle velocity vector and $\left(\sigma_{x x}, \sigma_{y y}, \sigma_{x y}\right)^{T}$ is the stress vector. At the initial time $t=t_{0} \geq 0$, we set the initial data

$$
\left(\begin{array}{c}
v_{x}\left(x, y, t=t_{0}\right) \\
v_{y}\left(x, y, t=t_{0}\right) \\
\sigma_{x x}\left(x, y, t=t_{0}\right) \\
\sigma_{y y}\left(x, y, t=t_{0}\right) \\
\sigma_{x y}\left(x, y, t=t_{0}\right)
\end{array}\right)=\left(\begin{array}{c}
v_{x}^{0}(x, y) \\
v_{y}^{0}(x, y) \\
\sigma_{x x}^{0}(x, y) \\
\sigma_{y y}^{0}(x, y) \\
\sigma_{x y}^{0}(x, y)
\end{array}\right) .
$$

Here, $\rho$ is the density and the compliance matrix is $\mathbf{S}=\mathbf{C}^{-1}$, where $\mathbf{C}=\mathbf{C}^{T}>0$ is the stiffness matrix. Since the stiffness matrix is symmetric positive definite, it also follows that the compliance matrix is symmetric positive definite, $\mathbf{S}=\mathbf{S}^{T}>0$. In an isotropic medium the stiffness matrix is given by

$$
\mathbf{C}=\left(\begin{array}{ccc}
2 \mu+\lambda & \lambda & 0 \\
\lambda & 2 \mu+\lambda & 0 \\
0 & 0 & \mu
\end{array}\right) .
$$

Here, $\lambda$ and $\mu$ are the first and the second Lamé parameters. The eigenvalues of $\mathbf{C}$ are $\mu, 2 \mu, 2(\mu+\lambda)$. For all $\mu>0$ and $\lambda>-\mu$, the eigenvalues of $\mathbf{C}$ are strictly positive. The elastic wave equation supports two families of waves, the $\mathrm{P}$-wave and the $\mathrm{S}$-wave, with the corresponding wave speeds $c_{p}$ and $c_{s}$ defined by

$$
c_{p}=\sqrt{\frac{2 \mu+\lambda}{\rho}}, \quad c_{s}=\sqrt{\frac{\mu}{\rho}} .
$$

In this work, we will consider constant elastic coefficients and scale time with $c_{p}$ such that density is unity $\rho \equiv 1$. Then we can introduce the non-dimensional parameter $\gamma=c_{s} / c_{p}$, the ratio of the wave-speeds. The case $\gamma \rightarrow 0$ corresponds to the incompressible or acoustic limit. Note also that the scaling results to a non-dimensional stiffness matrix

$$
\widetilde{\mathbf{C}}=\left(\begin{array}{ccc}
1 & 1-2 \gamma^{2} & 0 \\
1-2 \gamma^{2} & 1 & 0 \\
0 & 0 & \gamma^{2}
\end{array}\right)
$$

The eigenvalues of the scaled stiffness matrix $\widetilde{\mathbf{C}}$ are $\gamma^{2}, 2 \gamma^{2}, 2\left(1-\gamma^{2}\right)$. For all $\mu>0, \lambda \geq 0$, the velocity ratio satisfies $0<\gamma \leq \sqrt{2} / 2$. Nevertheless, in general the condition on $\gamma$ for well-posedness is $0<\gamma^{2}<1$.

For completeness, we close the edges of the rectangle with the general linear boundary conditions

$$
\frac{1-r_{x}^{(x)}}{2} v_{x} \pm \frac{1+r_{x}^{(x)}}{2} \sigma_{x x}=0, \quad \frac{1-r_{y}^{(x)}}{2} \gamma v_{y} \pm \frac{1+r_{y}^{(x)}}{2} \sigma_{x y}=0, \quad \text { at } \quad x= \pm x_{0}
$$




$$
\frac{1-r_{x}^{(y)}}{2} \gamma v_{x} \pm \frac{1+r_{x}^{(y)}}{2} \sigma_{x y}=0, \quad \frac{1-r_{y}^{(y)}}{2} v_{y} \pm \frac{1+r_{y}^{(y)}}{2} \sigma_{y y}=0, \quad \text { at } \quad y= \pm y_{0}
$$

The non-dimensional real constants $r_{x}^{(x)}, r_{y}^{(x)}, r_{x}^{(y)}, r_{y}^{(y)}$ with $\left|r_{x}^{(x)}\right| \leq 1,\left|r_{y}^{(x)}\right| \leq 1,\left|r_{x}^{(y)}\right| \leq 1,\left|r_{y}^{(y)}\right| \leq 1$ are reflection coefficients. For particular sets of the reflection coefficients we have

$$
\begin{aligned}
\text { clamped wall: } & r_{x}^{(x)}=r_{y}^{(x)}=-1, \quad r_{x}^{(y)}=r_{y}^{(y)}=-1, \\
\text { free surface: } & r_{x}^{(x)}=r_{y}^{(x)}=1, \quad r_{x}^{(y)}=r_{y}^{(y)}=1 \\
\text { characteristic: } & r_{x}^{(x)}=r_{y}^{(x)}=0, \quad r_{x}^{(y)}=r_{y}^{(y)}=0 .
\end{aligned}
$$

Note that with $\left|r_{x}^{(x)}\right|=1,\left|r_{y}^{(x)}\right|=1,\left|r_{x}^{(y)}\right|=1,\left|r_{y}^{(y)}\right|=1$, then from (6)-(7) we have

$$
\begin{aligned}
& v_{x} \sigma_{x x}=v_{y} \sigma_{x y}=0, \quad \text { at } \quad x= \pm x_{0} \\
& v_{x} \sigma_{x y}=v_{y} \sigma_{y y}=0, \quad \text { at } \quad y= \pm y_{0} .
\end{aligned}
$$

If $\left|r_{x}^{(x)}\right|<1,\left|r_{y}^{(x)}\right|<1,\left|r_{x}^{(y)}\right|<1,\left|r_{y}^{(y)}\right|<1$, then from (6)-(7) we have

$$
\begin{aligned}
& v_{x} \sigma_{x x}=\mp \frac{1-r_{x}^{(x)}}{1+r_{x}^{(x)}} v_{x}^{2}=\mp \frac{1+r_{x}^{(x)}}{1-r_{x}^{(x)}} \sigma_{x x}^{2}, \quad v_{y} \sigma_{x y}=\mp \gamma \frac{1-r_{y}^{(x)}}{1+r_{y}^{(x)}} v_{y}^{2}=\mp \frac{1}{\gamma} \frac{1+r_{y}^{(x)}}{1-r_{y}^{(x)}} \sigma_{x y}^{2}, \quad \text { at } \quad x= \pm x_{0}, \\
& v_{x} \sigma_{x y}=\mp \gamma \frac{1-r_{x}^{(y)}}{1+r_{x}^{(y)}} v_{x}^{2}=\mp \frac{1}{\gamma} \frac{1+r_{x}^{(y)}}{1-r_{x}^{(y)}} \sigma_{x y}^{2}, \quad v_{y} \sigma_{y y}=\mp \frac{1-r_{y}^{(y)}}{1+r_{y}^{(y)}} v_{y}^{2}=\mp \frac{1+r_{y}^{(y)}}{1-r_{y}^{(y)}} \sigma_{y y}^{2}, \quad \text { at } \quad x= \pm y_{0} .
\end{aligned}
$$

Using standard energy methods and the expressions (9)-(10), it can be shown that the scaled elastic wave equation (1) with the boundary conditions (6)-(7) satisfies

$$
\mathrm{E}_{\gamma}(t) \leq \mathrm{E}_{\gamma}(0), \quad \forall t \geq 0
$$

Here

$$
\mathrm{E}_{\gamma}(t)=\int_{\Omega}\left(\frac{1}{2}\left(v_{x}^{2}+v_{y}^{2}\right)+\frac{1}{2}\left(\begin{array}{c}
\sigma_{x x} \\
\sigma_{y y} \\
\sigma_{x y}
\end{array}\right)^{T} \widetilde{\mathbf{S}}\left(\begin{array}{c}
\sigma_{x x} \\
\sigma_{y y} \\
\sigma_{x y}
\end{array}\right)\right) d x d y
$$

with $\widetilde{\mathbf{S}}=\widetilde{\mathbf{C}}^{-1}$, is the scaled elastic energy. In the acoustic limit $\gamma \rightarrow 0, \widetilde{\mathbf{C}}$ is no longer invertible so the energy (12) is not appropriate. However, if $\gamma=0$ then $\sigma_{x y} \equiv \sigma_{x y}^{0}$, and $\sigma_{x x}=\sigma_{y y}=-P$ denotes the acoustic pressure. We can define the energy

$$
\mathrm{E}_{0}(t)=\int_{\Omega} \frac{1}{2}\left(v_{x}^{2}+v_{y}^{2}+P^{2}\right) d x d y
$$

We also have

$$
\mathrm{E}_{0}(t) \leq \mathrm{E}_{0}(0)
$$

In this paper we will exclusively consider the PML in an elastic solid, with $\gamma>0$. The analysis in [11] can be easily adapted to study the stability of the PML in acoustic media, with $\gamma=0$.

\subsection{PML for the elastic wave equation}

The PML for the elastic wave equation in first order form is well established, see for instance [3] for the modal PML and [2] for the split field PML. As above, we consider an isotropic homogeneous elastic rectangular block. To begin with, let the Laplace transform of $\mathbf{v}(x, y, t)$ be defined by

$$
\widehat{\mathbf{v}}(x, y, s)=\int_{0}^{\infty} e^{-s t} \mathbf{v}(x, y, t) \mathrm{dt}, \quad s=a+i b, \quad \Re s=a>0 .
$$

We consider a setup where the PML is included in both the $x$-direction and $y$-direction. Take the Laplace transform of the elastic wave equation (1) in time. The PML can be constructed direction-by-direction using $\partial / \partial x \rightarrow 1 / S_{x} \partial / \partial x$, $\partial / \partial y \rightarrow 1 / S_{y} \partial / \partial y$, for the vertical and horizontal layers, respectively. Here

$$
S_{x}=1+\frac{d_{x}(x)}{s+\alpha}, \quad S_{y}=1+\frac{d_{y}(y)}{s+\alpha},
$$


are the complex PML metrics, with $s$ denoting the Laplace dual time variable. The time-dependent modal PML is defined by

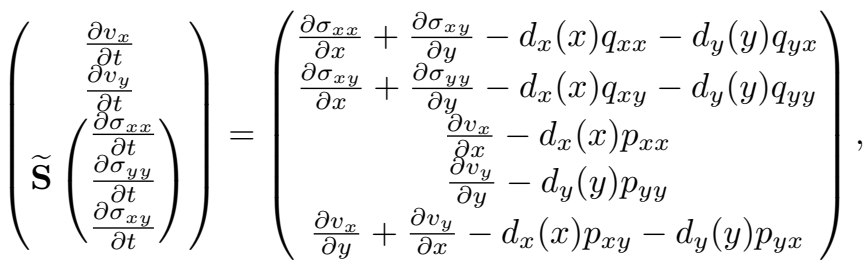

$$
\begin{aligned}
& \left(\begin{array}{l}
\frac{\partial p_{x x}}{\partial t} \\
\frac{\partial p_{x y}}{\partial t} \\
\frac{\partial q_{x x}}{\partial t} \\
\frac{\partial x_{x y}}{\partial t}
\end{array}\right)=\left(\begin{array}{c}
\frac{\partial v_{x}}{\partial x}-\left(d_{x}(x)+\alpha\right) p_{x x} \\
\frac{\partial v_{y}}{\partial x}-\left(d_{x}(x)+\alpha\right) p_{x y} \\
\frac{\partial \sigma x x}{\partial x}-\left(d_{x}(x)+\alpha\right) q_{x x} \\
\frac{\partial \sigma_{x y}}{\partial x}-\left(d_{x}(x)+\alpha\right) q_{x y}
\end{array}\right), \\
& \left(\begin{array}{l}
\frac{\partial p_{y x}}{\partial t} \\
\frac{\partial p_{y y}}{\partial t} \\
\frac{\partial q_{y x}}{\partial t} \\
\frac{\partial q_{y y}}{\partial t}
\end{array}\right)=\left(\begin{array}{c}
\frac{\partial v_{x}}{\partial y}-\left(d_{y}(y)+\alpha\right) p_{y x} \\
\frac{\partial v_{y}}{\partial y}-\left(d_{y}(y)+\alpha\right) p_{y y} \\
\frac{\partial \sigma_{x y}}{\partial y}-\left(d_{y}(y)+\alpha\right) q_{y x} \\
\frac{\partial \sigma_{y y}}{\partial y}-\left(d_{y}(y)+\alpha\right) q_{y y}
\end{array}\right) .
\end{aligned}
$$

Here, $d_{x}(x), d_{y}(y) \geq 0$ are the damping functions and $\alpha \geq 0$ is the complex frequency shift (CFS) [7]. The unknowns $\left(p_{x x}, p_{x y}\right)^{T},\left(p_{y x}, p_{y y}\right)^{T},\left(q_{x x}, q_{x y}\right)^{T},\left(q_{y x}, q_{y y}\right)^{T}$ are auxiliary variables introduced to localize the PML in time. The PML (17)-(19) is the same as the PML derived in [3]. It is possible to choose auxiliary variables differently in the Laplace space yielding a different system of PDEs in the physical space. However, all resulting PMLs can be shown to be linearly equivalent to (17)-(19). We will initialize the PML with zero initial data and terminate the PML (17)-(19) with the linear boundary conditions (6)-(7). Note that the damping $d_{x}, d_{y}$ and auxiliary functions vanish almost everywhere except in the layers defining the PML. In particular the $x$-dependent PML damping function $d_{x}(x)$ is nonzero, $d_{x}(x)>0, d_{y}(y)=0$, only in the vertical PML layers truncating the left and right edges of the computational domain and the $y$-dependent PML damping function $d_{y}(y)$ is nonzero, $d_{x}(x)=0, d_{y}(y)>0$, in the horizontal PML layers truncating the bottom and top edges of the computational domain. There are also corner regions where both damping functions are simultaneously nonzero, $d_{x}(x)>0, d_{y}(y)>0$.

Without damping, $d_{x}(x) \equiv 0, d_{y}(y) \equiv 0$, we recover the elastic wave equation (1), which satisfies the energy estimate (11). However, the energy estimate is not applicable to the PML, (17)-(19) with $d_{x}(x)>0$ or $d_{y}(y)>0$.

\section{Stability}

Here, we perform the stability analysis of the PML. The analysis focuses on the constant coefficient problem. To succeed, we will divide the problem into 3: a) a vertical PML layer with $d_{x}>0, d_{y}=0$, b) horizontal PML layer with $d_{x}=0, d_{y}>0$, and c) a PML corner region with $d_{x}>0, d_{y}>0$. The stability analysis of each PML layer can be performed using normal mode analysis by considering separately the corresponding half-plane problems with $d_{x}>0, d_{y}=0$ or $d_{x}=0, d_{y}>0$, subject to the boundary conditions (6) and (7) respectively. We will focus on a vertical PML layer with $d_{x}>0, d_{y}=0$ and analyze the corresponding half-plane PML problems subject to the boundary conditions (6) and (7). A similar analysis is valid for the horizontal PML layer, with $d_{x}=0, d_{y}>0$. We will also show that the PML (17)-(19) with the boundary conditions (6)-(7) ensure a stable and robust treatment of PML corners, when $d_{x}=d_{y}>0$.

\subsection{The vertical PML layer problem}

Consider now the vertical PML layer with constant damping $d_{x}=d>0, d_{y}=0$. The auxiliary differential equation (19) decouples from the modified elastic wave equation (17). Thus we consider (17)-(18) with $d_{x}=d>0, d_{y}=0$ subject to the boundary conditions (6)-(7). The stability analysis of the IBVP (17)-(18) with (6)-(7) is not as straightforward. Following [11], we simplify further by splitting the problem into: 1) a Cauchy problem (17)-(18) in $-\infty<x<\infty$, $-\infty<y<\infty$, with no boundary conditions, 2) a lower half-plane problem (17)-(18) in $-\infty<x<\infty,-\infty<y \leq y_{0}$ with the boundary condition (7) at $y=y_{0}$, and 3) a left half-plane problem (17)-(18) in $-\infty<x \leq x_{0},-\infty<y<\infty$ with the boundary condition (6) at $x=x_{0}$. Each of the three problems can be analyzed separately.

For the Cauchy PML problem the stability of the PML is rather well understood for various classes of problems, see [3] for the modal PML and [2] for the split field PML. The Cauchy problem will not be discussed further. 
For the lower half-plane PML problem it is straight forward to adapt the theory developed in [10, 4] for second order system. In isotropic elastic materials, the results of $[10,4]$ show that the PML IBVP subject to the physical boundary condition (7) at $y=y_{0}$, is asymptotically stable.

There is no stability theory yet for the left half-plane PML problem, with the boundary condition (6) terminating the PML at $x=x_{0}$. Here, we develop a stability theory for the left half-plane problem. In particular, we prove that the corresponding PML IBVP does not support nontrivial growing modes.

\subsubsection{A PML half plane problem with damping normal to the boundary}

We will now consider the left half-plane problem, that is the PML (17)-(18) in $-\infty<x \leq x_{0},-\infty<y<\infty$, with the boundary condition (6) at $x=x_{0}$. The ansatz

$$
\mathbf{U}(x, y, t)=e^{s t+i k_{y} y} \widehat{\mathbf{U}}(x),
$$

yields

$$
\left(S_{x} s\right)^{2} \widehat{\mathbf{v}}=\mathbf{A} \frac{\mathrm{d}^{2} \widehat{\mathbf{v}}}{\mathrm{d} x^{2}}+\left(i S_{x} k_{y}\right)^{2} \mathbf{B} \widehat{\mathbf{v}}+\left(i S_{x} k_{y}\right)\left(\mathbf{C}+\mathbf{C}^{T}\right) \frac{\mathrm{d} \widehat{\mathbf{v}}}{\mathrm{d} x}
$$

and

$$
\frac{1}{2 S_{x} s}\left(\left(S_{x} s\right)(\mathbf{I}-\mathbf{R}) \sqrt{\mathbf{A}} \widehat{\mathbf{v}}+(\mathbf{I}+\mathbf{R})\left(\mathbf{A} \frac{\mathrm{d} \widehat{\mathbf{v}}}{\mathrm{d} x}+i\left(S_{x} k_{y}\right) \mathbf{C} \widehat{\mathbf{v}}\right)\right)=0, \quad x=x_{0} .
$$

In (21)-(22) we have eliminated the stress fields and the auxiliary variables. Here, $S_{x}$ is the PML metric given by (16) and the coefficient matrices are given by

$$
\mathbf{A}=\left(\begin{array}{cc}
1 & 0 \\
0 & \gamma^{2}
\end{array}\right), \quad \sqrt{\mathbf{A}}=\left(\begin{array}{ll}
1 & 0 \\
0 & \gamma
\end{array}\right), \quad \mathbf{B}=\left(\begin{array}{cc}
\gamma^{2} & 0 \\
0 & 1
\end{array}\right), \quad \mathbf{C}=\left(\begin{array}{cc}
0 & 1-2 \gamma^{2} \\
\gamma^{2} & 0
\end{array}\right), \quad \mathbf{R}=\left(\begin{array}{cc}
r_{x}^{(x)} & 0 \\
0 & r_{y}^{(x)}
\end{array}\right), \quad \mathbf{I}=\left(\begin{array}{ll}
1 & 0 \\
0 & 1
\end{array}\right) .
$$

We will only consider $\left|r_{x}\right| \leq 1,\left|r_{y}\right| \leq 1$. Introducing $\widehat{s}=S_{x} s$ and $\widehat{k}_{y}=S_{x} k_{y}$ simplifies the notation, so that we have

$$
\widehat{s}^{2} \widehat{\mathbf{v}}=\mathbf{A} \frac{\mathrm{d}^{2} \widehat{\mathbf{v}}}{\mathrm{d} x^{2}}+\left(i \widehat{k}_{y}\right)^{2} \mathbf{B} \widehat{\mathbf{v}}+i \widehat{k}_{y}\left(\mathbf{C}+\mathbf{C}^{T}\right) \frac{\mathrm{d} \widehat{\mathbf{v}}}{\mathrm{d} x},
$$

with the boundary condition

$$
\frac{1}{2 \widehat{s}}\left(\widehat{s}(\mathbf{I}-\mathbf{R}) \sqrt{\mathbf{A}} \widehat{\mathbf{v}}+(\mathbf{I}+\mathbf{R})\left(\mathbf{A} \frac{\mathrm{d} \widehat{\mathbf{v}}}{\mathrm{d} x}+i \widehat{k}_{y} \mathbf{C} \widehat{\mathbf{v}}\right)\right)=0, \quad x=x_{0} .
$$

Note that the corresponding PML half-plane problem for the elastic wave equation in second order form yields the same system, (23) and (24). For the ordinary differential equation (23) we can construct modal solutions

$$
\widehat{\mathbf{v}}=\boldsymbol{\Phi} e^{\kappa x}
$$

Substituting (25) into (23) yields

$$
\left(\widehat{s}^{2} \mathrm{I}-\kappa^{2} \mathbf{A}+\widehat{k}_{y}^{2} \mathbf{B}-i \widehat{k}_{y} \kappa\left(\mathbf{C}+\mathbf{C}^{T}\right)\right) \boldsymbol{\Phi}=0,
$$

with all nontrivial solutions satisfying

$$
\operatorname{det}\left(\widehat{s}^{2} \mathrm{I}-\kappa^{2} \mathbf{A}+\widehat{k}_{y}^{2} \mathbf{B}-i \widehat{k}_{y} \kappa\left(\mathbf{C}+\mathbf{C}^{T}\right)\right)=0 .
$$

The characteristic equation (27) has four roots $\kappa= \pm \widehat{\kappa}_{1}, \kappa= \pm \widehat{\kappa}_{2}$, with the corresponding eigenvectors

$$
\boldsymbol{\Phi}_{1}^{(+)}=\left[\begin{array}{c}
\frac{-i \widehat{k}_{y}}{\widehat{\kappa}_{1}} \\
1
\end{array}\right], \quad \boldsymbol{\Phi}_{1}^{(-)}=\left[\begin{array}{c}
\frac{i \widehat{k}_{y}}{\widehat{\kappa}_{1}} \\
1
\end{array}\right], \quad \Phi_{2}^{(+)}=\left[\begin{array}{c}
\frac{\widehat{\kappa}_{2}}{i \widehat{k}_{y}} \\
1
\end{array}\right], \boldsymbol{\Phi}_{2}^{(-)}=\left[\begin{array}{c}
-\widehat{\kappa}_{2} \\
i \widehat{k}_{y} \\
1
\end{array}\right],
$$

where

$$
\widehat{\kappa}_{1}=\sqrt{\frac{\widehat{s}^{2}+\gamma^{2} \widehat{k}_{y}^{2}}{\gamma^{2}}}=S_{x} \sqrt{\frac{s^{2}+\gamma^{2} k_{y}^{2}}{\gamma^{2}}}, \quad \widehat{\kappa}_{2}=\sqrt{\widehat{s}^{2}+\widehat{k}_{y}^{2}}=S_{x} \sqrt{s^{2}+k_{y}^{2}} .
$$


Note in particular that since $\widehat{k}_{y}=S_{x} k_{y}$, the eigen-functions $\boldsymbol{\Phi}_{j}$ are independent of the PML parameter $S_{x}$. The general solution is

$$
\widehat{\mathbf{v}}=\theta_{1} \boldsymbol{\Phi}_{1}^{(+)} e^{\widehat{\kappa}_{1} x}+\beta_{1} \boldsymbol{\Phi}_{1}^{(-)} e^{-\widehat{\kappa}_{1} x}+\theta_{2} \boldsymbol{\Phi}_{2}^{(+)} e^{\widehat{\kappa}_{2} x}+\beta_{2} \boldsymbol{\Phi}_{2}^{(-)} e^{-\widehat{\kappa}_{2} x}
$$

It can be shown that for all $d, \alpha \geq 0, \Re s>0$ and $k_{y} \in \mathbb{R}$, we have $\Re \widehat{\kappa}_{1}, \Re \widehat{\kappa}_{2}>0$; see Corollary 1 in the Appendix. Thus boundedness of the solution at $x \rightarrow-\infty$ implies $\beta_{1}, \beta_{2}=0$. The parameters $\theta_{1}, \theta_{2}$ are determined by the boundary condition (24). Dropping the superscript $(+)$ and inserting (28) in (24) we obtain

$$
\frac{1}{2 \widehat{s}}\left(\widehat{s}(\mathbf{I}-\mathbf{R}) \sqrt{\mathbf{A}}\left(\theta_{1} \boldsymbol{\Phi}_{1}+\theta_{2} \boldsymbol{\Phi}_{2}\right)+(\mathbf{I}+\mathbf{R})\left(\mathbf{A}\left(\widehat{\kappa}_{1} \theta_{1} \boldsymbol{\Phi}_{1}+\widehat{\kappa}_{2} \theta_{2} \boldsymbol{\Phi}_{2}\right)+i \widehat{k}_{y} \mathbf{C}\left(\theta_{1} \boldsymbol{\Phi}_{1}+\theta_{2} \boldsymbol{\Phi}_{2}\right)\right)\right)=0 .
$$

Since $\widehat{s}=S_{x} s$, and $\widehat{\kappa}_{1}=S_{x} \kappa_{1}, \widehat{\kappa}_{2}=S_{x} \kappa_{2}, \widehat{k}_{y}=S_{x} k_{y}$ where

$$
\kappa_{1}=\sqrt{\frac{s^{2}+\gamma^{2} k_{y}^{2}}{\gamma^{2}}}, \quad \kappa_{2}=\sqrt{s^{2}+k_{y}^{2}},
$$

we have

$$
\frac{1}{2 s}\left(s(\mathbf{I}-\mathbf{R}) \sqrt{\mathbf{A}}\left(\theta_{1} \boldsymbol{\Phi}_{1}+\theta_{2} \boldsymbol{\Phi}_{2}\right)+(\mathbf{I}+\mathbf{R})\left(\mathbf{A}\left(\kappa_{1} \theta_{1} \boldsymbol{\Phi}_{1}+\kappa_{2} \theta_{2} \boldsymbol{\Phi}_{2}\right)+i k_{y} \mathbf{C}\left(\theta_{1} \boldsymbol{\Phi}_{1}+\theta_{2} \boldsymbol{\Phi}_{2}\right)\right)\right)=0 .
$$

It is important to note that the system of equations (30) does not depend on any of the PML parameters $d, \alpha$, and is identical to the corresponding equation for the undamped problem. This is unlike the corresponding equation for the lower half-plane problem, which depends on the PML parameters $d, \alpha$, see $[10,4]$. Thus, if the undamped left half-plane problem is stable, it follows that the PML IBVP is also stable.

We can now rewrite $(30)$ as

$$
\mathcal{K}\left(s, k_{y}, r_{x}^{(x)}, r_{y}^{(x)}\right)\left(\begin{array}{c}
\theta_{1} \\
\theta_{2}
\end{array}\right)=0,
$$

where $\mathcal{K}\left(s, k_{y}, r_{x}^{(x)}, r_{y}^{(x)}\right)$ is a $2 \times 2$ matrix. Stability can be characterized by the equation

$$
\mathcal{C}\left(s, k_{y}, r_{x}^{(x)}, r_{y}^{(x)}\right):=\operatorname{det}\left(\mathcal{K}\left(s, k_{y}, r_{x}^{(x)}, r_{y}^{(x)}\right)\right)=0 .
$$

If there is a root with $\Re s>0$ then all corresponding IBVPs (damped/undamped, first/second order formulation) are unstable in every sense.

In addition, if there are purely imaginary roots $s=i \beta$ and corresponding nontrivial modes $\left(\theta_{1}, \theta_{2}\right) \neq 0$, all corresponding IBVPs will support boundary wave modes. These boundary wave modes move orthogonally to the PML damping direction and are not damped by the PML. Since the determinant is independent of the PML parameters we can use results for the undamped IBVP to predict the existence of boundary modes along the outer boundary of a PML for different boundary conditions.

Theorem 1. Consider the constant constant coefficient left half plane PML problem with $d_{x}=d>0, d_{y}=0$ subject to the boundary condition (6) at $x=x_{0}$ with $\left|r_{x}^{(x)}\right| \leq 1,\left|r_{y}^{(x)}\right| \leq 1$. The IBVP has no nontrivial solution of the form (25) with $\Re s>0$.

Proof:

Since the elastic wave equation (1) with the boundary condition (6) at $x=x_{0}$ satisfy the energy estimate (11) we must have $\Re s \leq 0$, for all $k_{y} \in \mathbb{R}$. Otherwise if $\Re s>0$, then the energy will grow for some solutions, which is also in contradiction with the energy estimate (11).

We consider in more detail three commonly used boundary conditions

$$
\begin{aligned}
\text { clamped wall: } & r_{x}^{(x)}=r_{y}^{(x)}=-1, \quad \widehat{v}_{x}=0, \quad \widehat{v}_{y}=0, \\
\text { free surface: } & r_{x}^{(x)}=r_{y}^{(x)}=1, \quad \widehat{\sigma}_{x x}=0, \quad \widehat{\sigma}_{x y}=0, \\
\text { characteristic: } & r_{x}^{(x)}=r_{y}^{(x)}=0, \quad \frac{1}{2} \widehat{v}_{x}+\frac{1}{2} \widehat{\sigma}_{x x}=0, \quad \frac{1}{2} \gamma \widehat{v}_{y}+\frac{1}{2} \widehat{\sigma}_{x y}=0 .
\end{aligned}
$$

The determinant $\mathcal{C}\left(s, k_{y}, r_{x}^{(x)}, r_{y}^{(x)}\right)$ is defined in the positive complex plane, $\Re s \geq 0$, but it can be extended to the negative complex plane, $\Re s<0$. We summarize the results as follows: 
Theorem 2. Consider the determinant equation $\mathcal{C}\left(s, k_{y}, r_{x}^{(x)}, r_{y}^{(x)}\right)=0$ defined by (31).

a) clamped wall: There are no roots of $\mathcal{C}\left(s, k_{y},-1,-1\right)=0$ for all $s \in \mathbb{C}$, thus implying $\theta_{1}=\theta_{2}=0$.

b) free surface: Any root $s$ of $\mathcal{C}\left(s, k_{y}, 1,1\right)=0$ must be purely imaginary $s=i \beta$, with $\beta \in \mathbb{R}$.

c) characteristic: Any root s of $\mathcal{C}\left(s, k_{y}, 0,0\right)=0$ must satisfy $\Re s<0$.

\section{Proof:}

See Appendix B.

The the clamped boundary condition (32a) does not support boundary wave modes. The purely imaginary roots of the free surface boundary condition correspond to Rayleigh wave modes propagating on the surface of an elastic solid without decay. When a PML is closed by the free surface boundary condition (32b) at the outer edge, Rayleigh wave modes are supported, and propagate perpendicularly to the PML damping direction without decay along the outer edge. This is in contrast to the free surface boundary conditions at $y=y_{0}$, where all Rayleigh wave modes are damped by the PML as they move along the boundary in the PML damping direction; see [10, 4]. Only temporally decaying boundary modes can be supported by the characteristic boundary condition (32c).

\subsubsection{One space dimensional energy estimate in the Laplace space}

In the previous subsection we established, using modal analysis, the temporal stability of the continuous PML problem (17)-(18) subject to the boundary conditions (6) terminating the PML at $x=x_{0}$. The remaining challenge lies in constructing numerical approximations and ensuring numerical stability. Extending this modal analysis to the discrete setting is possible, but non-trivial in all but the most simplified cases. This motivates the development of an energy estimate that can be used to guide the numerical implementation of boundary conditions. The power of the energy estimate is that we can construct stable numerical discretization by mimicking this continuous energy estimate in a discrete setting.

To begin, take the Laplace transform in time of the PML equations (17)-(19) and the boundary conditions (6)-(7), we have

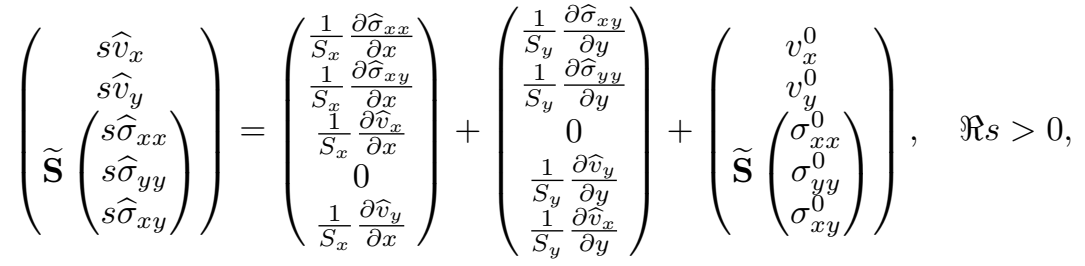

$$
\begin{aligned}
& \frac{1-r_{x}^{(x)}}{2} \widehat{v}_{x} \pm \frac{1+r_{x}^{(x)}}{2} \widehat{\sigma}_{x x}=0, \quad \frac{1-r_{y}^{(x)}}{2} \gamma \widehat{v}_{y} \pm \frac{1+r_{y}^{(x)}}{2} \widehat{\sigma}_{x y}=0, \quad \text { at } \quad x= \pm x_{0}, \\
& \frac{1-r_{x}^{(y)}}{2} \gamma \widehat{v}_{x} \pm \frac{1+r_{x}^{(y)}}{2} \widehat{\sigma}_{x y}=0, \quad \frac{1-r_{y}^{(y)}}{2} \widehat{v}_{y} \pm \frac{1+r_{y}^{(y)}}{2} \widehat{\sigma}_{y y}=0, \quad \text { at } \quad y= \pm y_{0} .
\end{aligned}
$$

Here $\mathbf{U}^{0}=\left(v_{x}^{0}, v_{y}^{0}, \sigma_{x x}^{0}, \sigma_{y y}^{0}, \sigma_{x y}^{0}\right)^{T}$ denotes the initial data. In (33) we have eliminated the auxiliary variables. Introduce $\widehat{\mathbf{U}}=\left(\widehat{v}_{x}, \widehat{v}_{y}, \widehat{\sigma}_{x x}, \widehat{\sigma}_{y y}, \widehat{\sigma}_{x y}\right)^{T}$, and define the weighted scalar product

$$
\left(\widehat{\mathbf{U}}, \mathbf{U}^{0}\right)_{\gamma}=\int_{\Omega}\left(\frac{1}{2}\left(\widehat{v}_{x}^{*} v_{x}^{0}+\widehat{v}_{y}^{*} v_{y}^{0}\right)+\frac{1}{2}\left(\begin{array}{c}
\widehat{\sigma}_{x x} \\
\widehat{\sigma}_{y y} \\
\widehat{\sigma}_{x y}
\end{array}\right)^{H} \widetilde{\mathbf{S}}\left(\begin{array}{c}
\sigma_{x x}^{0} \\
\sigma_{y y}^{0} \\
\sigma_{x y}^{0}
\end{array}\right)\right) d x d y,
$$

and the corresponding energy

$$
\widehat{\mathrm{E}}_{\gamma}(s) \equiv(\widehat{\mathbf{U}}, \widehat{\mathbf{U}})_{\gamma}=\int_{\Omega}\left(\frac{1}{2}\left(\left|\widehat{v}_{x}\right|^{2}+\left|\widehat{v}_{y}\right|^{2}\right)+\frac{1}{2}\left(\begin{array}{c}
\widehat{\sigma}_{x x} \\
\widehat{\sigma}_{y y} \\
\widehat{\sigma}_{x y}
\end{array}\right)^{H} \widetilde{\mathbf{S}}\left(\begin{array}{c}
\widehat{\sigma}_{x x} \\
\widehat{\sigma}_{y y} \\
\widehat{\sigma}_{x y}
\end{array}\right)\right) d x d y
$$

Here, $v^{*}$ denotes the complex conjugate of $v, \widehat{\mathbf{v}}^{H}$ denotes the complex conjugate transpose of $\widehat{\mathbf{v}}$ and $\widetilde{\mathbf{S}}=\widetilde{\mathbf{C}}^{-1}$, with $\widetilde{\mathbf{C}}$ defined in (5). 
Theorem 3. Consider the constant coefficient PML equation in the Laplace space (33) with $d_{x}(x) \equiv d>0, d_{y}(y) \equiv 0$ and $\Re s>0$. The solution of the corresponding one space dimensional problem with vanishing derivatives in the $y$-direction and the boundary condition (34) at $x= \pm x_{0}$, satisfies

$$
\Re\left(s S_{x}\right) \widehat{\mathrm{E}}_{\gamma}(s)=\Re\left(\widehat{\mathbf{U}}, S_{x} \widehat{\mathbf{U}}^{0}\right)_{\gamma}+\mathrm{BT} .
$$

Here $\Re\left(s S_{x}\right)>0$ and $\mathrm{BT}=\Re\left(\left.\int_{y=-y_{0}}^{y=y_{0}}\left(\widehat{v}_{x}^{*} \widehat{\sigma}_{x x}+\widehat{v}_{y}^{*} \widehat{\sigma}_{x y}\right)\right|_{x=-x_{0}} ^{x=x_{0}} d y\right)$. If $\left|r_{x}^{(x)}\right|<1,\left|r_{x}^{(x)}\right|<1$, then $\mathrm{BT}<0$. With strict equality $\left|r_{x}^{(x)}\right|=\left|r_{x}^{(x)}\right|=1$ we have $\mathrm{BT}=0$.

\section{Proof:}

Let $d_{x}(x) \equiv d>0, d_{y}(y) \equiv 0$ and $\partial / \partial y \equiv 0$ in (33), and multiply equation (33) by $S_{x} \widehat{\mathbf{U}}^{H}$ from the left. Adding the conjugate of the product and integrating over the whole domain gives

$$
\Re\left(s S_{x}\right) \widehat{\mathrm{E}}_{\gamma}(s)=\Re\left(\widehat{\mathbf{U}}, S_{x} \widehat{\mathbf{U}}^{0}\right)_{\gamma}+\mathrm{BT} .
$$

For $\alpha=0$, we have $s S_{x}=s+d(x)$, implying $\Re\left(s S_{x}\right)>0$, for all $\Re s=a>0$ and $d(x) \geq 0$. If $\alpha>0$ it can also be shown that $\Re\left(s S_{x}\right)>0$, for all $\Re s=a>0$, see equation (A.5) in the Appendix. Here, BT are boundary terms given by

$$
\mathrm{BT}=\Re\left(\left.\int_{y=-y_{0}}^{y=y_{0}}\left(\widehat{v}_{x}^{*} \widehat{\sigma}_{x x}+\widehat{v}_{y}^{*} \widehat{\sigma}_{x y}\right)\right|_{x=-x_{0}} ^{x=x_{0}} d y\right) .
$$

By applying the boundary condition (34) it follows that for $\left|r_{x}^{(x)}\right|=\left|r_{y}^{(x)}\right|=1$ we have

$$
\mathrm{BT}=0 .
$$

For $\left|r_{x}^{(x)}\right|<1,\left|r_{x}^{(x)}\right|<1$ we have

$$
\mathrm{BT}=-\int_{y=-y_{0}}^{y=y_{0}}\left(\frac{1-r_{x}^{(x)}}{1+r_{x}^{(x)}}\left|\widehat{v}_{x}\right|^{2}+\gamma \frac{1-r_{y}^{(x)}}{1+r_{y}^{(x)}}\left|\widehat{v}_{y}\right|^{2}\right)_{x=-x_{0}} d y-\int_{y=-y_{0}}^{y=y_{0}}\left(\frac{1-r_{x}^{(x)}}{1+r_{x}^{(x)}}\left|\widehat{v}_{x}\right|^{2}+\gamma \frac{1-r_{y}^{(x)}}{1+r_{y}^{(x)}}\left|\widehat{v}_{y}\right|^{2}\right)_{x=x_{0}} d y \leq 0,
$$

or equivalently

$$
\mathrm{BT}=-\int_{y=-y_{0}}^{y=y_{0}}\left(\frac{1+r_{x}^{(x)}}{1-r_{x}^{(x)}}\left|\widehat{\sigma}_{x x}\right|^{2}+\frac{1}{\gamma} \frac{1+r_{y}^{(x)}}{1-r_{y}^{(x)}}\left|\widehat{\sigma}_{x y}\right|^{2}\right)_{x=-x_{0}} d y-\int_{y=-y_{0}}^{y=y_{0}}\left(\frac{1+r_{x}^{(x)}}{1-r_{x}^{(x)}}\left|\widehat{\sigma}_{x x}\right|^{2}+\frac{1}{\gamma} \frac{1+r_{y}^{(x)}}{1-r_{y}^{(x)}}\left|\widehat{\sigma}_{x y}\right|^{2}\right)_{x=x_{0}} d y \leq 0 .
$$

The proof of the theorem is complete.

While the boundary terms (42) or (43) will dissipate of energy, the boundary terms (41) is non-dissipative. Note that since the boundary terms BT are always zero or negative we can use Cauchy-Schwartz inequality to obtain a strict bound on the solution in terms of the initial data

$$
\sqrt{\widehat{\mathrm{E}}_{\gamma}(s)} \leq \frac{\left|S_{x}\right|}{\Re\left(s S_{x}\right)} \sqrt{\mathrm{E}_{\gamma}^{0}}
$$

Here, $\mathrm{E}_{\gamma}^{0}$ denotes the elastic energy for the initial data. However, the energy estimate (44) is not sharp. In particular, unlike the energy equation (39), the energy estimate (44) does not give any information on how energy is dissipated by the boundary terms.

Remark 1. It is important to note that a similar modal analysis in subsection 3.1 .1 and the energy estimates (39) and (44) are also valid for the horizontal PML layer, that is when $d_{x}=0, d_{y}=d>0$.

\subsection{The PML corner problem}

Consider now a constant coefficient PML in the corner region, (17)-(19) with $d_{x}=d_{y}=d>0$. Then, the PML metrics are identical $S_{y}=S_{x}$. As above, the application of standard energy methods yields the energy equation

$$
\Re\left(s S_{x}\right) \widehat{\mathrm{E}}_{\gamma}(s)=\Re\left(\widehat{\mathbf{U}}, S_{x} \widehat{\mathbf{U}}^{0}\right)_{\gamma}+\mathrm{BT}_{x}+\mathrm{BT}_{y} .
$$

Here, $\widehat{\mathrm{E}}_{\gamma}(s)$ is the elastic energy defined in (37) and the boundary terms are given by

$$
\mathrm{BT}_{x}=\Re\left(\left.\int_{y=-y_{0}}^{y=y_{0}}\left(\widehat{v}_{x}^{*} \widehat{\sigma}_{x x}+\widehat{v}_{y}^{*} \widehat{\sigma}_{x y}\right)\right|_{x=-x_{0}} ^{x=x_{0}} d y\right), \quad \mathrm{BT}_{y}=\Re\left(\left.\int_{x=-x_{0}}^{x=x_{0}}\left(\widehat{v}_{x}^{*} \widehat{\sigma}_{x y}+\widehat{v}_{y}^{*} \widehat{\sigma}_{y y}\right)\right|_{y=-y_{0}} ^{y=y_{0}} d x\right) .
$$

By (41)-(43), we know that the imposition of the boundary conditions (34)-(35) must satisfy $\mathrm{BT}_{x} \leq 0, \mathrm{BT}_{y} \leq 0$ for all $\left|r_{x}^{(x)}\right| \leq 1,\left|r_{y}^{(x)}\right| \leq 1,\left|r_{x}^{(y)}\right| \leq 1,\left|r_{y}^{(y)}\right| \leq 1$. As in the 1D case the boundary terms vanish if the corresponding reflection coefficients have unit absolute values. As before we classify the boundary procedure as non-dissipative or dissipative depending on wether the boundary terms vanish or are negative definite. Finally we note that the PML corner problem with $d_{x}=d_{y}=d>0$ also satisfies the energy estimate (44). 


\section{Numerical approximations}

In this section, we introduce several discrete approximations. We will use SBP finite difference operators to approximate all spatial derivatives in (17)-(19) and impose boundary conditions (6)-(7) weakly using penalties. In order to ensure numerical stability we will choose penalty parameters such that we can derive discrete energy estimates analogous to the continuous energy estimate (39) and (45).

\subsection{Spatial discrete operators}

To begin with, consider the uniform discretization of the interval, $-x_{0} \leq x \leq x_{0}$, with $N_{x}$ number of grid points and the uniform spatial step $h_{x}>0$

$$
x_{i}=-x_{0}+(i-1) h_{x}, \quad i=1,2, \ldots, N_{x}, \quad h_{x}=\frac{2 x_{0}}{N_{x}-1} .
$$

Let $D_{x}$ be a differentiation matrix approximating the first derivative, $D_{x} \approx \partial / \partial x$, on the uniform grid (47). The matrix $D_{x}$ is an SBP operator (in one space dimension), if the following properties hold

$$
D_{x}=\mathrm{H}_{x}^{-1} Q_{x}, \quad Q_{x}^{T}+Q_{x}=E_{R x}-E_{L x}, \quad \mathrm{H}_{x}=\mathrm{H}_{x}^{T}>0 .
$$

Here, $E_{L x}=\operatorname{diag}(1,0,0, \ldots, 0), E_{R x}=\operatorname{diag}(0,0,0, \ldots, 1)$ pick out the left and right boundary values. The matrix $Q_{x}$ is almost skew-symmetric. The matrix $\mathrm{H}_{x}$ is diagonal with positive entries $h_{i i}^{(x)}>0$, thus defining a discrete norm. Note in particular that $\mathrm{H}_{x}$ defines a quadrature rule.

From the SBP property (48) we also have

$$
D_{x}=\mathrm{H}_{x}^{-1} Q_{x}=\mathrm{H}_{x}^{-1}\left(-\left(\mathrm{H}_{x}^{-1} Q_{x}\right)^{T} \mathrm{H}_{x}+E_{R x}-E_{L x}\right)=-\mathrm{H}_{x}^{-1} D_{x}^{T} \mathrm{H}_{x}+\mathrm{H}_{x}^{-1}\left(E_{R x}-E_{L x}\right) .
$$

The most important property of the SBP finite difference operator $D_{x}$ defined in (48) is that it mimics the integrationby-parts property of the continuous operator. This is crucial in order to prove numerical stability. The SBP operator used in this study can be found in [26].

We discretize the rectangular domain, in the $x$ - and $y$-directions, using $N_{x}, N_{y}$ grid points, respectively, with uniform spatial steps $h_{x}, h_{y}>0$. A two space dimensional scalar grid function $\mathbf{v}$ is stacked as a vector of length $N_{x} N_{y}$,

$$
\mathbf{v}=\left(v_{11}, v_{12}, \ldots, v_{N_{x} N_{y}}\right)^{T} .
$$

Spatial operators in higher space dimensions are derived using the Kronecker products,

$$
\begin{aligned}
& \mathbf{D}_{x}=\left(D_{x} \otimes I_{y}\right), \quad \mathbf{D}_{y}=\left(I_{x} \otimes D_{y}\right), \quad \mathbf{H}_{x}=\left(H_{x} \otimes I_{y}\right), \quad \mathbf{H}_{y}=\left(I_{x} \otimes H_{y}\right), \\
& \mathbf{E}_{x}^{+}=\left(E_{R x} \otimes I_{y}\right), \quad \mathbf{E}_{y}^{+}=\left(I_{x} \otimes E_{R y}\right), \quad \mathbf{E}_{x}^{-}=\left(E_{L x} \otimes I_{y}\right), \quad \mathbf{E}_{y}^{-}=\left(I_{x} \otimes E_{L y}\right) .
\end{aligned}
$$

Here, $D_{x}, D_{y}$ are one space dimensional SBP operators with diagonal norms $H_{x}, H_{y}$. The matrices $E_{L x}, E_{L y}, E_{R x}, E_{R y}$ are boundary operators, and $I_{x}, I_{y}$ are identity matrices of sizes corresponding to the number of grid points $N_{x}, N_{y}$.

\subsection{Stable discretization of the PML IBVP}

The discrete approximation of the PML equations (17)-(19) using SBP operators with SAT enforcements of the boundary conditions (6)-(7) can be written as

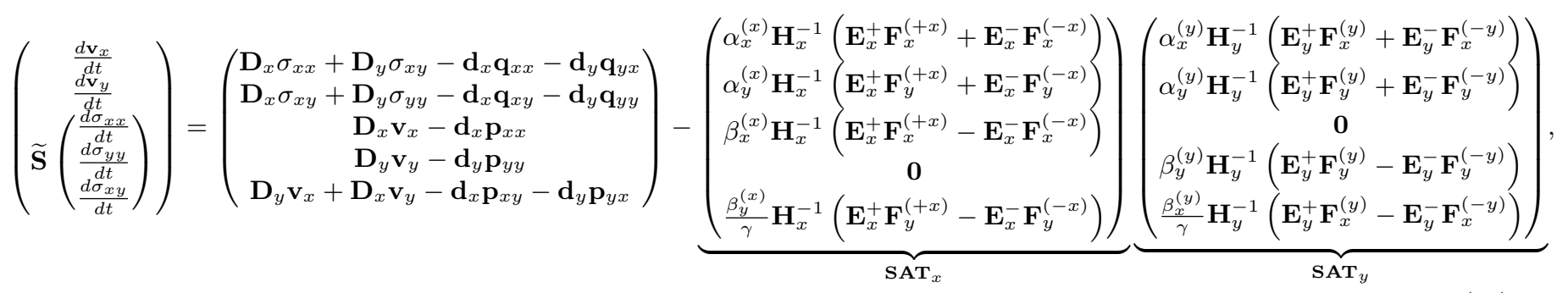

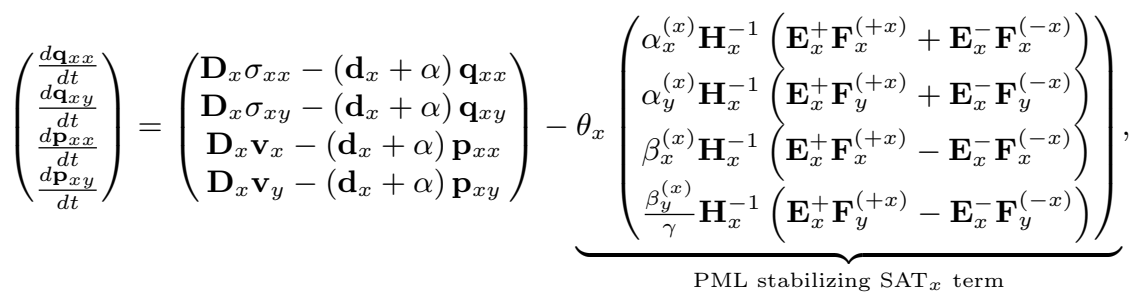




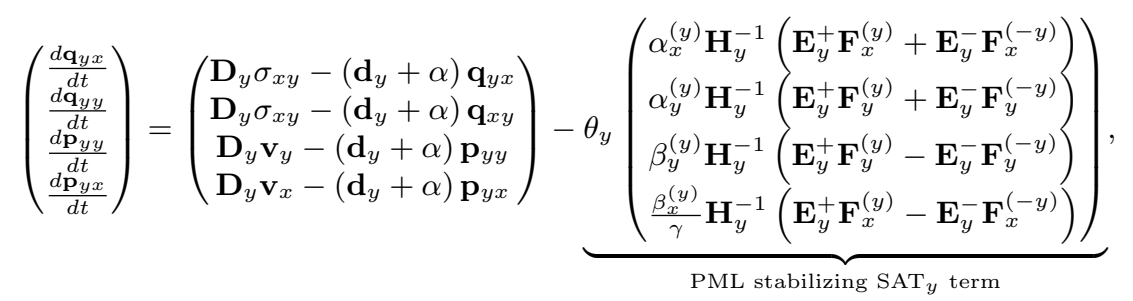

where $\mathbf{F}^{( \pm x)}, \mathbf{F}^{( \pm y)}$ are the SAT forcing terms defined by

$$
\begin{aligned}
& \mathbf{F}_{x}^{( \pm x)}:=\frac{1-r_{x}^{(x)}}{2} \mathbf{v}_{x} \pm \frac{1+r_{x}^{(x)}}{2} \sigma_{x x}, \quad \mathbf{F}_{y}^{( \pm x)}:=\frac{1-r_{y}^{(x)}}{2} \gamma \mathbf{v}_{y} \pm \frac{1+r_{y}^{(x)}}{2} \sigma_{x y}, \quad \text { at } \quad x= \pm x_{0}, \\
& \mathbf{F}_{x}^{( \pm y)}:=\frac{1-r_{x}^{(y)}}{2} \gamma \mathbf{v}_{x} \pm \frac{1+r_{x}^{(y)}}{2} \sigma_{x y}, \quad \mathbf{F}_{y}^{( \pm y)}:=\frac{1-r_{y}^{(y)}}{2} \mathbf{v}_{y} \pm \frac{1+r_{y}^{(y)}}{2} \sigma_{y y}, \quad \text { at } \quad y= \pm y_{0} .
\end{aligned}
$$

Note that the matrices $\mathbf{E}_{x}^{( \pm)}, \mathbf{E}_{y}^{( \pm)}$project the SAT forcing on the boundaries, and if the boundary conditions (6)-(7) are satisfied exactly then $\mathbf{F}^{( \pm x)} \equiv 0$ and $\mathbf{F}^{( \pm y)} \equiv 0$. The dimensionless real numbers $\alpha_{x}^{(x)}, \alpha_{y}^{(x)}, \beta_{x}^{(x)}, \beta_{y}^{(x)}, \theta_{x}$ and $\alpha_{x}^{(y)}, \alpha_{y}^{(y)}, \beta_{x}^{(y)}, \beta_{y}^{(y)}, \theta_{y}$ are penalty parameters determined by requiring stability. Note also that if $\theta_{x} \neq 0, \theta_{y} \neq 0$, the numerical boundary conditions are extended to the auxiliary differential equations. Often, numerical stability is proven in the absence of the PML, when $d_{y}(y)=d_{x}(x)=0$, and numerical boundary procedures are not extended to the auxiliary differential equation yielding the natural choice $\theta_{x}=\theta_{y}=0$. However, we will show that the penalty parameter $\theta_{x}=\theta_{y}=1$ is necessary for numerical stability when the PML is present, that is when $d_{x}(x) \neq 0, d_{y}(y) \neq 0$.

Below, we investigate numerical stability of the discrete approximations (50)-(52). We will consider first the undamped problem with $d_{x}(x) \equiv 0, d_{y}(y) \equiv 0$ and proceed later to the PML problem with $d_{x}(x) \geq 0, d_{y}(y) \geq 0$.

\subsection{Stability analysis for the undamped discrete problem}

We will begin with the undamped case, $d_{x}(x) \equiv 0, d_{y}(y) \equiv 0$. Then the auxiliary variables $\mathbf{p}$, q decouple completely from the discrete elastic wave equation (50). We approximate the elastic energy (12) by a quadrature rule

$$
\mathcal{E}_{\gamma h}(t)=\sum_{j=1}^{N_{y}} \sum_{i=1}^{N_{x}}\left(\frac{1}{2}\left(v_{x i j}^{2}+v_{y i j}^{2}\right)+\frac{1}{2}\left(\begin{array}{c}
\sigma_{x x i j} \\
\sigma_{y y i j} \\
\sigma_{x y i j}
\end{array}\right)^{T} \widetilde{\mathbf{S}}_{i j}\left(\begin{array}{c}
\sigma_{x x i j} \\
\sigma_{y y i j} \\
\sigma_{x y i j}
\end{array}\right)\right) h_{i i}^{(x)} h_{j j}^{(y)} .
$$

Note that for all SBP operators considered in this study, the quadrature weights are positive $h_{i i}^{(x)}, h_{j j}^{(y)},>0$. Therefore, the discrete quantity $\mathcal{E}_{\gamma h}(t)$ defined in (55) is a discrete elastic energy. If the discrete solutions satisfy $\mathcal{E}_{\gamma h}(t) \leq \mathcal{E}_{\gamma h}(0)$ for all $t \geq 0$, then we say that the numerical approximation is asymptotically stable.

Here we consider three separate penalty formulations for the undamped equations. Using standard energy analysis, the following can be shown to hold true (see [27] for details):

- Penalize all equations: Consider $\left|r_{x}^{(x)}\right| \leq 1,\left|r_{y}^{(x)}\right| \leq 1,\left|r_{x}^{(y)}\right| \leq 1,\left|r_{y}^{(y)}\right| \leq 1$. If $\alpha_{x}^{(x)}=\alpha_{y}^{(x)}=1, \quad \beta_{x}^{(x)}=\beta_{y}^{(x)}=1$, $\alpha_{x}^{(y)}=\alpha_{y}^{(y)}=1, \quad \beta_{x}^{(y)}=\beta_{y}^{(y)}=1$, then

$$
\begin{aligned}
\frac{d}{d t} \mathcal{E}_{\gamma h}(t)= & -\frac{1}{2} \sum_{j=1}^{N_{y}}\left(\left(1-r_{x}^{(x)}\right) v_{x 1 j}^{2}+\left(1-r_{y}^{(x)}\right) \gamma v_{y 1 j}^{2}+\left(1+r_{x}^{(x)}\right) \sigma_{x x 1 j}^{2}+\frac{\left(1+r_{y}^{(x)}\right)}{\gamma} \sigma_{x y 1 j}^{2}\right) h_{j j}^{(y)} \\
& -\frac{1}{2} \sum_{j=1}^{N_{y}}\left(\left(1-r_{x}^{(x)}\right) v_{x N_{x} j}^{2}+\left(1-r_{y}^{(x)}\right) \gamma v_{y N_{x} j}^{2}+\left(1+r_{x}^{(x)}\right) \sigma_{x x N_{x} j}^{2}+\frac{\left(1+r_{y}^{(x)}\right)}{\gamma} \sigma_{x y N_{x} j}^{2}\right) h_{j j}^{(y)} \\
& -\frac{1}{2} \sum_{i=1}^{N_{x}}\left(\left(1-r_{x}^{(y)}\right) \gamma v_{x i 1}^{2}+\left(1-r_{y}^{(y)}\right) v_{y i 1}^{2}+\frac{\left(1+r_{x}^{(y)}\right)}{\gamma} \sigma_{x y i 1}^{2}+\left(1+r_{y}^{(y)}\right) \sigma_{y y i 1}^{2}\right) h_{i i}^{(x)} \\
& -\frac{1}{2} \sum_{i=1}^{N_{x}}\left(\left(1-r_{x}^{(y)}\right) \gamma v_{x i N_{y}}^{2}+\left(1-r_{y}^{(y)}\right) v_{y i N_{y}}^{2}+\frac{\left(1+r_{x}^{(y)}\right)}{\gamma} \sigma_{x y i N_{y}}^{2}+\left(1+r_{y}^{(y)}\right) \sigma_{y y i N_{y}}^{2}\right) h_{i i}^{(x)}
\end{aligned}
$$

- Penalize the velocity equations only: Let $\beta_{x}^{(x)}=\beta_{y}^{(x)}=\beta_{x}^{(y)}=\beta_{y}^{(y)}=0$.

- A free-surface boundary: Consider $r_{x}^{(x)}=r_{y}^{(x)}=1, r_{x}^{(y)}=r_{y}^{(y)}=1$. If $\alpha_{x}^{(x)}=\alpha_{y}^{(x)}=1, \alpha_{x}^{(y)}=\alpha_{y}^{(y)}=1$, then the solutions satisfy the energy conservation equation

$$
\frac{d}{d t} \mathcal{E}_{\gamma h}(t)=0
$$


- A characteristic boundary: Consider $r_{x}^{(x)}=r_{y}^{(x)}=0, r_{x}^{(y)}=r_{y}^{(y)}=0$. If $\alpha_{x}^{(x)}=\alpha_{y}^{(x)}=2, \alpha_{x}^{(y)}=\alpha_{y}^{(y)}=2$, then the solutions satisfy the energy equation

$$
\begin{aligned}
\frac{d}{d t} \mathcal{E}_{\gamma h}(t)= & -\sum_{j=1}^{N_{y}}\left(v_{x 1 j}^{2}+\gamma v_{y 1 j}^{2}\right) h_{j j}^{(y)}-\sum_{j=1}^{N_{y}}\left(v_{x N_{x} j}^{2}+\gamma v_{y N_{x} j}^{2}\right) h_{j j}^{(y)} \\
& -\sum_{i=1}^{N_{x}}\left(\gamma v_{x i 1}^{2}+v_{y i 1}^{2}\right) h_{i i}^{(x)}-\sum_{i=1}^{N_{x}}\left(\gamma v_{x i N_{y}}^{2}+v_{y i N_{y}}^{2}\right) h_{i i}^{(x)}
\end{aligned}
$$

- Penalize the stress equations only: Let $\alpha_{x}^{(x)}=\alpha_{y}^{(x)}=\alpha_{x}^{(y)}=\alpha_{y}^{(y)}=0$.

- A clamped wall: Consider $r_{x}^{(x)}=r_{y}^{(x)}=-1, r_{x}^{(y)}=r_{y}^{(y)}=-1$. If $\beta_{x}^{(x)}=\beta_{y}^{(x)}=1, \beta_{x}^{(y)}=\beta_{y}^{(y)}=1$, then the solutions satisfy the energy conservation equation

$$
\frac{d}{d t} \mathcal{E}_{\gamma h}(t)=0
$$

- A characteristic boundary: Consider $r_{x}^{(x)}=r_{y}^{(x)}=0, r_{x}^{(y)}=r_{y}^{(y)}=0$. If $\beta_{x}^{(x)}=\beta_{y}^{(x)}=2, \beta_{x}^{(y)}=\beta_{y}^{(y)}=2$, then the solutions satisfy the energy equation

$$
\begin{aligned}
\frac{d}{d t} \mathcal{E}_{\gamma h}(t)= & -\sum_{j=1}^{N_{y}}\left(\sigma_{x x 1 j}^{2}+\frac{1}{\gamma} \sigma_{x y 1 j}^{2}\right) h_{j j}^{(y)}-\sum_{j=1}^{N_{y}}\left(\sigma_{x x N_{x} j}^{2}+\frac{1}{\gamma} \sigma_{x y N_{x} j}^{2}\right) h_{j j}^{(y)} \\
& -\sum_{i=1}^{N_{x}}\left(\frac{1}{\gamma} \sigma_{x y i 1}^{2}+\sigma_{y y i 1}^{2}\right) h_{i i}^{(x)}-\sum_{i=1}^{N_{x}}\left(\frac{1}{\gamma} \sigma_{x y i N_{y}}^{2}+\sigma_{y y i N_{y}}^{2}\right) h_{i i}^{(x)} .
\end{aligned}
$$

If we penalize all the equations with appropriate penalty weights as above, then the numerical boundary procedure is asymptotically stable and dissipative for all $\left|r_{x}^{(x)}\right| \leq 1,\left|r_{y}^{(x)}\right| \leq 1,\left|r_{x}^{(y)}\right| \leq 1,\left|r_{y}^{(y)}\right| \leq 1$. Also of importance is that the numerical boundary procedure is dissipative even if the boundary condition, with $\left|r_{x}^{(x)}\right|=1,\left|r_{y}^{(x)}\right|=1,\left|r_{x}^{(y)}\right|=1,\left|r_{y}^{(y)}\right|=1$, is non-dissipative in the continuous setting. Since the SAT imposition of boundary conditions is a consistent numerical procedure, the numerical dissipation will vanish in the limit of mesh refinement. When we penalize the velocity equations only or the stress equations only with appropriate penalty weights as above, we obtain discrete energy estimates which are in full accordance with the continuous energy estimates. In particular, the energy equations (57) or (59) demonstrate that the numerical treatments of the free surface boundary condition or the clamped wall boundary condition is asymptotically stable and non-dissipative. It is also noteworthy that the characteristic boundary conditions is asymptotically stable and dissipative in all above cases since there is energy dissipation in the continuous problem.

When the damping is absent $d_{x}(x) \equiv 0, d_{y}(y) \equiv 0$, we conclude that the above penalty parameters result in asymptotically stable discrete approximations. Note also that the energy estimates are independent of the penalty parameters $\theta_{x}, \theta_{x}$. However when the damping is present $d_{x}(x) \neq 0$ or $d_{y}(y) \neq 0$, the story is different.

\subsection{Stability of the discrete PML}

As in the continuous case we can not derive discrete energy estimates for the time dependent discrete PML problem. We will instead take the Laplace transform in time and choose penalties such that discrete energy estimates, analogous to (39), can be derived. Taking the Laplace transform, in time, of the semi-discrete constant coefficient problem (50)-(52) gives

$$
\begin{aligned}
& \left(\begin{array}{c}
s \widehat{\mathbf{v}}_{x} \\
s \widehat{\mathbf{v}}_{y} \\
\widetilde{\mathbf{S}}\left(\begin{array}{c}
s \widehat{\sigma}_{x x} \\
s \widehat{\sigma}_{y y} \\
s \widehat{\sigma}_{x y}
\end{array}\right)=\left(\begin{array}{c}
\frac{1}{S_{x}} \mathbf{D}_{x} \widehat{\sigma}_{x x}+\frac{1}{S_{y}} \mathbf{D}_{y} \widehat{\sigma}_{x y}-\frac{\alpha_{x}^{(x)}}{S_{x}} \mathbf{H}_{x}^{-1}\left(\mathbf{E}_{x}^{+} \widehat{\mathbf{F}}_{x}^{(+x)}+\mathbf{E}_{x}^{-} \widehat{\mathbf{F}}_{x}^{(-x)}\right)-\frac{\alpha_{x}^{(y)}}{S_{y}} \mathbf{H}_{y}^{-1}\left(\mathbf{E}_{y}^{+} \widehat{\mathbf{F}}_{x}^{(y)}+\mathbf{E}_{y}^{-} \widehat{\mathbf{F}}_{x}^{(-y)}\right) \\
\frac{1}{S_{x}} \mathbf{D}_{x} \widehat{\sigma}_{x y}+\frac{1}{S_{y}} \mathbf{D}_{y} \widehat{\sigma}_{y y}-\frac{\alpha_{y}^{(x)}}{S_{x}} \mathbf{H}_{x}^{-1}\left(\mathbf{E}_{x}^{+} \widehat{\mathbf{F}}_{y}^{(+x)}+\mathbf{E}_{x}^{-} \widehat{\mathbf{F}}_{y}^{(-x)}\right)-\frac{\alpha_{y}^{(y)}}{S_{y}} \mathbf{H}_{y}^{-1}\left(\mathbf{E}_{y}^{+} \widehat{\mathbf{F}}_{y}^{(y)}+\mathbf{E}_{y}^{-} \widehat{\mathbf{F}}_{y}^{(-y)}\right) \\
\frac{1}{S_{x}} \mathbf{D}_{x} \mathbf{v}_{x}-\frac{\beta_{x}^{(x)}}{S_{x}} \mathbf{H}_{x}^{-1}\left(\mathbf{E}_{x}^{+} \widehat{\mathbf{F}}_{x}^{(+x)}-\mathbf{E}_{x}^{-} \widehat{\mathbf{F}}_{x}^{(-x)}\right) \\
\frac{1}{S_{y}} \mathbf{D}_{y} \widehat{\mathbf{v}}_{y}-\frac{\beta_{y}^{(y)}}{S_{y}} \mathbf{H}_{y}^{-1}\left(\mathbf{E}_{y}^{+} \widehat{\mathbf{F}}_{y}^{(y)}-\mathbf{E}_{y}^{-} \widehat{\mathbf{F}}_{y}^{(-y)}\right) \\
\mathbf{v}_{x}^{0} \\
\mathbf{v}_{y}^{0} \\
\bar{S}_{y} \mathbf{D}_{y} \widehat{\mathbf{v}}_{x}+\frac{1}{S_{x}} \mathbf{D}_{x} \widehat{\mathbf{v}}_{y}-\frac{\beta_{y}^{(x)}}{\gamma S_{x}} \mathbf{H}_{x}^{-1}\left(\mathbf{E}_{x}^{+} \widehat{\mathbf{F}}_{y}^{(+x)}+\mathbf{E}_{x}^{-} \widehat{\mathbf{F}}_{y}^{(-x)}\right)-\frac{\beta_{x}^{(y)}}{\gamma S_{y}} \mathbf{H}_{y}^{-1}\left(\mathbf{E}_{y}^{+} \widehat{\mathbf{F}}_{x}^{(y)}+\mathbf{E}_{y}^{-} \widehat{\mathbf{F}}_{x}^{(-y)}\right)
\end{array}\right)\left(\begin{array}{l}
\left.\sigma_{x x}^{0}\right) \\
\sigma_{y y}^{0} \\
\sigma_{x y}^{0}
\end{array}\right)
\end{array}\right)
\end{aligned}
$$

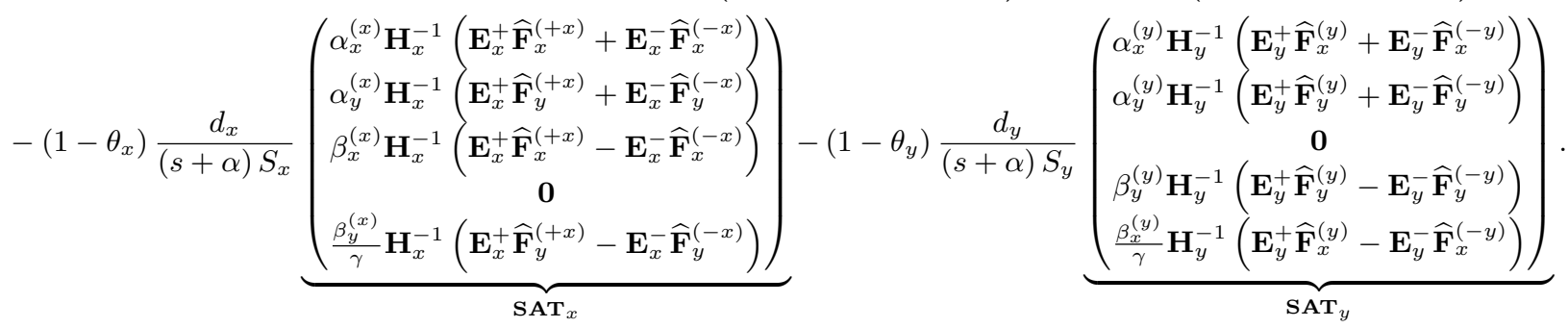


We set the penalty parameter $\theta_{x}=\theta_{y}=1$, extending the numerical boundary conditions to the auxiliary differential equations. The last two terms in the right hand side of (61) vanish. We approximate the scalar product (36) and the elastic energy (37) by a quadrature rule

$$
\begin{gathered}
\left\langle\widehat{\mathbf{U}}, \mathbf{U}^{0}\right\rangle_{\gamma h}(s)=\sum_{j=1}^{N_{y}} \sum_{i=1}^{N_{x}}\left(\frac{1}{2}\left(\widehat{v}_{x i j}^{*} \widehat{v}_{x i j}^{0}+\widehat{v}_{y i j}^{*} \widehat{v}_{y i j}^{0}\right)+\frac{1}{2}\left(\begin{array}{c}
\widehat{\sigma}_{x x i j} \\
\widehat{\sigma}_{y y i j} \\
\widehat{\sigma}_{x y i j}
\end{array}\right)^{H} \widetilde{\mathbf{S}}_{i j}\left(\begin{array}{c}
\widehat{\sigma}_{x x i j}^{0} \\
\widehat{\sigma}_{y y i j}^{0} \\
\widehat{\sigma}_{x y i j}^{0}
\end{array}\right)\right) h_{i i}^{(x)} h_{j j}^{(y)}, \\
\widehat{\mathcal{E}}_{\gamma h}(s) \equiv\langle\widehat{\mathbf{U}}, \widehat{\mathbf{U}}\rangle_{\gamma h}(s)=\sum_{j=1}^{N_{y}} \sum_{i=1}^{N_{x}}\left(\frac{1}{2}\left(\left|\widehat{v}_{x i j}\right|^{2}+\left|\widehat{v}_{y i j}\right|^{2}\right)+\frac{1}{2}\left(\begin{array}{l}
\widehat{\sigma}_{x x i j} \\
\widehat{\sigma}_{y y i j} \\
\widehat{\sigma}_{x y i j}
\end{array}\right)^{H} \widetilde{\mathbf{S}}_{i j}\left(\begin{array}{l}
\widehat{\sigma}_{x x i j} \\
\widehat{\sigma}_{y y i j} \\
\widehat{\sigma}_{x y i j}
\end{array}\right)\right) h_{i i}^{(x)} h_{j j}^{(y)} .
\end{gathered}
$$

The quantity $\widehat{\mathcal{E}}_{\gamma h}(s)$ defined above is strictly positive if $\widehat{\mathbf{U}}$ does not vanish identically.

\subsubsection{The discrete PML strip problem}

Let $d_{x}(x) \equiv d>0, d_{y}(y) \equiv 0$ in $(61)$. Consider the corresponding 1D problem and ignore the boundary terms at $y= \pm y_{0}$, we arrive at the discrete energy estimate

$$
\Re\left(s S_{x}\right) \widehat{\mathcal{E}}_{\gamma h}(s)=\Re\left\langle\widehat{\mathbf{U}}, S_{x} \mathbf{U}^{0}\right\rangle_{\gamma h}(s)+\mathcal{B} \mathcal{T}_{n u m} .
$$

Here, $\mathcal{B} \mathcal{T}_{\text {num }}$ is the contribution from the boundary, and is given by (65)-(69) for different combinations of boundary conditions and penalty parameters. If the numerical boundary procedure is stable then we say that the boundary terms must be zero $\mathcal{B} \mathcal{T}_{\text {num }}=0$ or negative $\mathcal{B} \mathcal{T}_{\text {num }}<0$. In particular that if $\mathcal{B T}_{\text {num }}=0$ then the boundary procedure is non-dissipative, and if $\mathcal{B T}_{\text {num }} \leq 0$ then the numerical boundary procedure is called dissipative. Note that the numerical boundary procedure can be dissipative even if the corresponding boundary condition in the continuous setting is non-dissipative.

Theorem 4. Consider the semi-discrete PML equation in the Laplace space $(61)$, and let $d_{x}(x) \equiv d>0, d_{y}(y) \equiv 0$ with $D_{y}=0$. We have

$$
\Re\left(s S_{x}\right) \widehat{\mathcal{E}}_{\gamma h}(s)=\Re\left\langle\widehat{\mathbf{U}}, S_{x} \mathbf{U}^{0}\right\rangle_{\gamma h}(s)+\mathcal{B} \mathcal{T}_{\text {num }} .
$$

For specific values of the penalty parameters the following are true:

- Penalize all equations: Consider $\left|r_{x}^{(x)}\right| \leq 1,\left|r_{y}^{(x)}\right| \leq 1,\left|r_{x}^{(y)}\right| \leq 1,\left|r_{y}^{(y)}\right| \leq 1$. If $\alpha_{x}^{(x)}=\alpha_{y}^{(x)}=1, \quad \beta_{x}^{(x)}=\beta_{y}^{(x)}=1$, $\alpha_{x}^{(y)}=\alpha_{y}^{(y)}=1, \quad \beta_{x}^{(y)}=\beta_{y}^{(y)}=1$, and $\theta_{x}=1$, the boundary term is

$$
\begin{aligned}
\mathcal{B} \mathcal{T}_{\text {num }} & =-\frac{1}{2} \sum_{j=1}^{N_{y}}\left(\left(1-r_{x}^{(x)}\right)\left|\widehat{v}_{x 1 j}\right|^{2}+\left(1-r_{y}^{(x)}\right) \gamma\left|\widehat{v}_{y 1 j}\right|^{2}+\left(1+r_{x}^{(x)}\right)\left|\widehat{\sigma}_{x x 1 j}\right|^{2}+\frac{\left(1+r_{y}^{(x)}\right)}{\gamma}\left|\widehat{\sigma}_{x y 1 j}\right|^{2}\right) h_{j j}^{(y)} \\
& -\frac{1}{2} \sum_{j=1}^{N_{y}}\left(\left(1-r_{x}^{(x)}\right)\left|\widehat{v}_{x N_{x} j}\right|^{2}+\left(1-r_{y}^{(x)}\right) \gamma\left|\widehat{v}_{y N_{x} j}\right|^{2}+\left(1+r_{x}^{(x)}\right)\left|\widehat{\sigma}_{x x N_{x} j}\right|^{2}+\frac{\left(1+r_{y}^{(x)}\right)}{\gamma}\left|\widehat{\sigma}_{x y N_{x} j}\right|^{2}\right) h_{j j}^{(y)} .
\end{aligned}
$$

- Penalize the velocity equations only. Let $\beta_{x}^{(x)}=\beta_{y}^{(x)}=0$ :

- A free-surface boundary: Consider $r_{x}^{(x)}=r_{y}^{(x)}=1$. If $\alpha_{x}^{(x)}=\alpha_{y}^{(x)}=1, \theta_{x}=1$, then the boundary term is

$$
\mathcal{B} \mathcal{T}_{\text {num }}=0 .
$$

- A characteric boundary: Consider $r_{x}^{(x)}=r_{y}^{(x)}=0, r_{x}^{(y)}=r_{y}^{(y)}=0$. If $\alpha_{x}^{(x)}=\alpha_{y}^{(x)}=2, \alpha_{x}^{(y)}=\alpha_{y}^{(y)}=2, \theta_{x}=1$, then the boundary term is

$$
\mathcal{B} \mathcal{T}_{\text {num }}=-\sum_{j=1}^{N_{y}}\left(\left|\widehat{v}_{x 1 j}\right|^{2}+\gamma\left|\widehat{v}_{y 1 j}\right|^{2}\right) h_{j j}^{(y)}-\sum_{j=1}^{N_{y}}\left(\left|\widehat{v}_{x N_{x} j}\right|^{2}+\gamma\left|\widehat{v}_{y N_{x} j}\right|^{2}\right) h_{j j}^{(y)} .
$$

- Penalize the stress equations only. Let $\alpha_{x}^{(x)}=\alpha_{y}^{(x)}=0$ :

- A clamped wall (penalize the stress equations only): Consider $r_{x}^{(x)}=r_{y}^{(x)}=-1$. If $\beta_{x}^{(x)}=\beta_{y}^{(x)}=1, \theta_{x}=1$, then the boundary term is

$$
\mathcal{B T}_{\text {num }}=0 .
$$


- A characteristic boundary: Consider $r_{x}^{(x)}=r_{y}^{(x)}=0, r_{x}^{(y)}=r_{y}^{(y)}=0$. If $\beta_{x}^{(x)}=\beta_{y}^{(x)}=2, \beta_{x}^{(y)}=\beta_{y}^{(y)}=2, \theta_{x}=1$, then the boundary term is

$$
\mathcal{B} \mathcal{T}_{\text {num }}=-\sum_{j=1}^{N_{y}}\left(\left|\widehat{\sigma}_{x x 1 j}\right|^{2}+\frac{1}{\gamma}\left|\widehat{\sigma}_{x y 1 j}\right|^{2}\right) h_{j j}^{(y)}-\sum_{j=1}^{N_{y}}\left(\left|\widehat{\sigma}_{x x N_{x} j}\right|^{2}+\frac{1}{\gamma}\left|\widehat{\sigma}_{x y N_{x} j}\right|^{2}\right) h_{j j}^{(y)} .
$$

The estimates (65)-(69) are only possible with $\theta_{x}=1$. When $d \neq 0$ and $\theta_{x} \neq 1$, these estimates do not hold due to the boundary terms arising from the PML. Numerical experiments presented in the next section demonstrate that it is essential the estimates hold, also for two space dimensional problems. We also note that in analogy with the previous section we can separate dissipative and non-dissipative boundary procedures. In equations (65), (67), (69) the boundary terms contribute to dissipation, and the corresponding numerical boundary procedures are called dissipative, while the numerical boundary procedures corresponding to (66), (68) are non-dissipative.

Numerical experiments performed in the next section also demonstrate that to guarantee long-time stability the numerical boundary treatment must be dissipative. As seen above, for a linear well-posed boundary condition terminating the PML, a dissipative numerical boundary procedure can be derived using the following approaches:

a) The continuous boundary condition is dissipative, as in the characteristic boundary condition (32c). All stable penalty parameters will result in a dissipative numerical boundary procedure.

b) The continuous boundary condition is non-dissipative, for example using the clamped wall (32a) or the free surface boundary condition (32b). These boundary conditions need extra care, because not all penalty parameters will result to dissipative numerical boundary procedures. See for examples the penalty parameters corresponding to (66) and (68). To ensure a dissipative numerical boundary procedure we must penalize all equations appropriately using for example the penalty parameters yielding (65).

\subsubsection{The discrete PML corner problem}

Consider now a the discrete PML (61) in the corner region with $d_{x}=d_{y}=d>0$ and $\theta_{x}=\theta_{y}=1$. For this case the PML complex metrics are identical $S_{y}=S_{x}$. As above, the application of standard energy methods yields the energy equation

$$
\Re\left(s S_{x}\right) \widehat{\mathcal{E}}_{\gamma h}(s)=\Re\left\langle\widehat{\mathbf{U}}, S_{x} \mathbf{U}^{0}\right\rangle_{\gamma h}(s)+\mathcal{B} \mathcal{T}_{\text {xnum }}+\mathcal{B} \mathcal{T}_{\text {ynum }}
$$

Here, $\mathcal{B T}_{\text {xnum }}, \mathcal{B T}_{\text {ynum }}$ are the corresponding boundary terms. With the specific penalty parameters as above the boundary terms are completely analogous to (65)-(69). It is therefore easy show that the boundary terms are negative semi-definite, that is $\mathcal{B} \mathcal{T}_{\text {xnum }} \leq 0, \mathcal{B} \mathcal{T}_{\text {ynum }} \leq 0$. The analysis here is restricted to $d_{x}=d_{y}$, however the numerical experiments presented in the next section demonstrate that the PML ensures a stable and robust treatment of PML corners with variable damping functions $0 \leq d_{x}(x) \neq d_{y}(y) \geq 0$.

\section{Numerical experiments}

In this section, we present numerical experiments. The experiments are designed to quantify numerical errors introduced by discretizing the PML as well as verify the stability analysis of the last section. We will first consider the vertical PML strip problem and proceed later to simulate a half-plane problem surrounded by the PML. The later situation involves both the vertical (with $d_{x}(x) \geq 0, d_{y}(y)=0$ ) and horizontal (with $\left.d_{x}(x)=0, d_{y}(y) \geq 0\right) \mathrm{PML}$ layers, and PML corners (with $d_{x}(x) \geq 0, d_{y}(y) \geq 0$ ) where both layers are simultaneously active.

\subsection{The vertical strip PML problem}

To begin with, consider a rectangular elastic solid with the dimension $(x, y)=[-50,50] \times[0,50]$. On the top surface of the elastic solid, at $y=50$, we set the free surface boundary conditions, with $r_{x}^{(y)}=r_{y}^{(y)}=1$ in $(7)$. The bottom of the solid, at $y=0$, is a fictional wall with $r_{x}^{(y)}=r_{y}^{(y)}=0$ in (7), that is the characteristic boundary condition. Both boundary conditions in the $y$-direction will be enforced weakly by penalizing the velocity equations only. It is also possible to use different sets of penalties as discussed in the last section. In the $x$-direction we introduce two additional layers, having $50 \leq|x| \leq 50+\delta$ in which the PML equations are solved. In order to complete the statement of the problem, at the edges of the PML, we set the well-posed boundary conditions (6) with $\left|r_{x}^{(x)}\right| \leq 1,\left|r_{y}^{(x)}\right| \leq 1$. We will vary the boundary parameters $\left|r_{x}^{(x)}\right| \leq 1,\left|r_{y}^{(x)}\right| \leq 1$ in the numerical experiments to determine optimal PML boundary closure. In particular we will consider separately, a free surface boundary condition with $r_{x}^{(x)}=r_{y}^{(x)}=1$, a clamped wall boundary condition with $r_{x}^{(x)}=r_{y}^{(x)}=-1$ and a characteristic boundary conditions with $r_{x}^{(x)}=r_{y}^{(x)}=0$. We will also consider different sets of penalty parameters presented in the previous section. 
To begin we set the initial condition

$$
v_{x}=v_{y}=e^{-\log (2) \frac{x^{2}+(y-25)^{2}}{9}},
$$

for the velocity fields, and zero initial condition for the stress fields and all auxiliary variables. The damping profile is a cubic monomial

$$
d(x)=\left\{\begin{array}{ccc}
0 & \text { if } & |x| \leq 50 \\
d_{0}\left(\frac{|x|-50}{\delta}\right)^{3} & \text { if } & |x| \geq 50
\end{array}\right.
$$

where $d_{0} \geq 0$ is the damping strength. We will use

$$
d_{0}=\frac{4 c_{p}}{2 \delta} \ln \frac{1}{\text { tol }}
$$

where $c_{p}$ denotes the P-wave speed, and tol is the magnitude of the relative PML error [12].

We discretize in space with a high order accurate SBP operator. The spatial operator has a sixth order accurate interior stencil and a third order accurate boundary closure, and thus yields a theoretical global fourth order accurate finite difference stencil [26]. All boundary conditions are enforced weakly using SAT, as described in the last section. Numerical approximation in time is obtained by the classical fourth order accurate Runge-Kutta scheme. The time step $k$ is given by

$$
k=\frac{0.5}{\sqrt{c_{p}^{2}+c_{s}^{2}}} h .
$$

We investigate numerically the stability of the PML. We consider an aluminum solid defined by $\gamma=0.4593$. The corresponding $\mathrm{P}$-wave and $\mathrm{S}$-wave velocities are given by $c_{p}=2.1772, c_{s}=1$. In the coming experiments we set the magnitude of the relative PML error tol $=10^{-4}$. We will use the complex frequency shift $\alpha=0.15$ in this experiment. In a practical calculation the PML width is considerably short, we therefore use $\delta=10$, leading to the damping strength $d_{0}=4.0106$. The width of the PML $\delta=10$ corresponds to $10 \%$ of the width of the computational domain. We will experiment with a free surface boundary condition, a clamped wall boundary condition, and a characteristic boundary condition to establish appropriate boundary conditions terminating the PML. We will also consider separately $\theta_{x}=0$ and $\theta_{x}=1$, and different penalty parameters in the experiments.

Set a uniform spatial step $h=1$ in both $x$ and $y$ axes. We run the simulation until $t=5000$, corresponding to 23959 time steps, and record the pointwise maximum norm of the velocity vector in the entire domain. In figure 2 , numerical results for $\theta_{x}=0$, using different penalty parameters are presented. Note that with $\theta_{x}=0$ numerical solutions grow for all choices of penalty parameters considered here. However, if the numerical boundary procedure is dissipative the numerical solution is stable over a short time but numerical growth occurs at a much later time. We also turned off the PML, $d(x) \equiv 0$, and run the same simulations as before. Numerical results are shown in figure 3. When the PML is absent, all solutions decay with time for all boundary parameters and all different choices of penalties.

From the previous section we know that the PML stabilizing SAT terms are essential for deriving an energy estimate already in one space dimension. Therefore we use $\theta_{x}=1$ in the next set of experiments, that is we include the PML stabilizing SAT term in the discrete auxiliary differential equations. Numerical results for this situation is presented in figure 4 . Observe that, if $\theta_{x}=1$ and the boundary condition or a numerical enforcement of the boundary condition is dissipative then the numerical solutions are long-time stable. However, if the boundary condition is non-dissipative and the corresponding discretization is also non-dissipative, then the numerical solution is not long-time stable, even with $\theta_{x}=1$.

These numerical results indicate that the PML stabilizing penalty suggested by the one dimensional analysis are necessary also for higher space dimensional problems. They also indicate that, to avoid growth one must additionally require a dissipative numerical boundary procedure. In isolation, neither of these will stabilize the PML, but together they will stabilize the PML.

In [6], numerical instabilities are seen to be more severe for small $\gamma>0$, nearly incompressible materials. In the next experiment we will demonstrate the stability of our method for several velocity ratio $\gamma$. We set the relative PML error tol $=10^{-4}$ and terminate the PML with the characteristic boundary conditions. Since the P-wave speed $c_{p}$ increase with decreasing $\gamma$ it also follows from (73) that the damping coefficient $d_{0}$ will increase with decreasing $\gamma$. For various velocity ratios $\gamma=0.1,0.2,0.3,0.4,0.5$ and the spatial step $h=1$, we compute the solution for a longer time, until $t=10000$. The snapshots of the solution at $t=5,20,40$ are shown in figure 5 for $\gamma=0.2,0.4$. Figure 5 shows how the initial pulse spreads, being reflected by the free-surface and the absorption of waves by the PML. In 


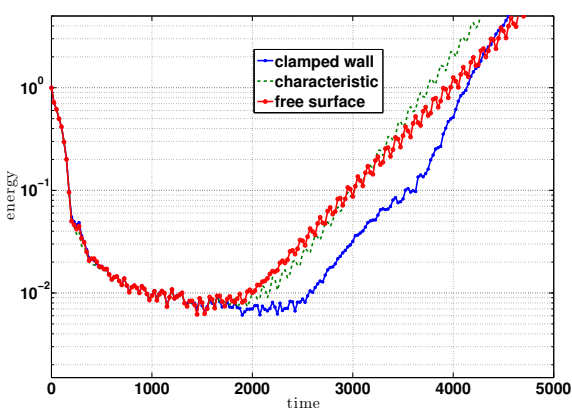

(a) Penalize all equations.

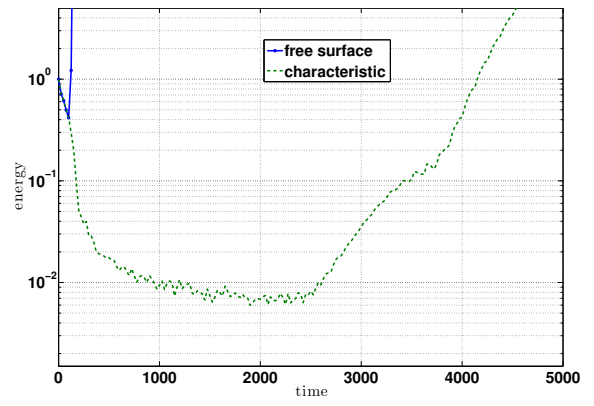

(b) Penalize velocity equations only.

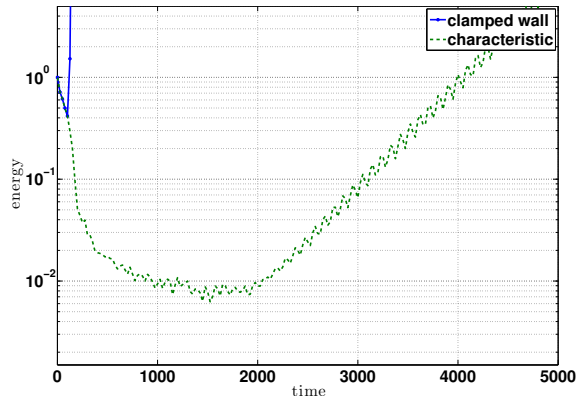

(c) Penalize stress equations only.

Figure 2: Time series of the $L_{2}$ norm of the velocity vector for the PML without the PML stabilizing penalty, $\theta_{x}=0$.

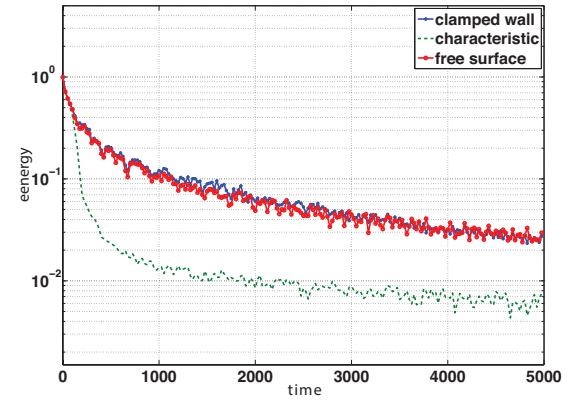

(a) Penalize all equations.

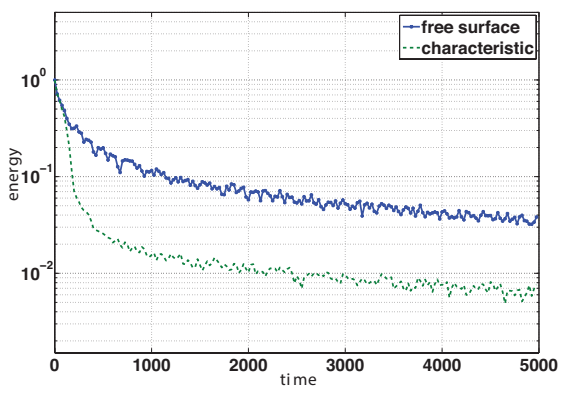

(b) Penalize velocity equations only.

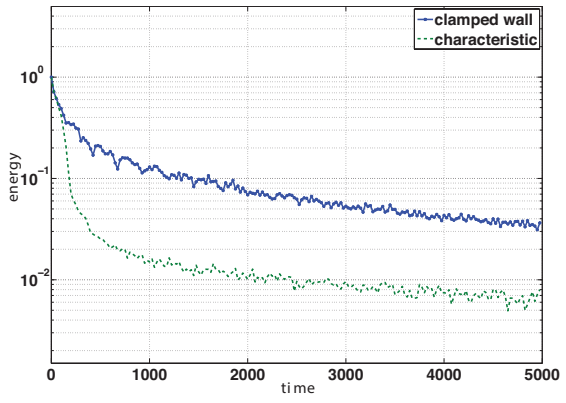

(c) Penalize stress equations only.

Figure 3: Time series of the $L_{2}$ norm of the velocity vector without the $P M L, d(x) \equiv 0$.

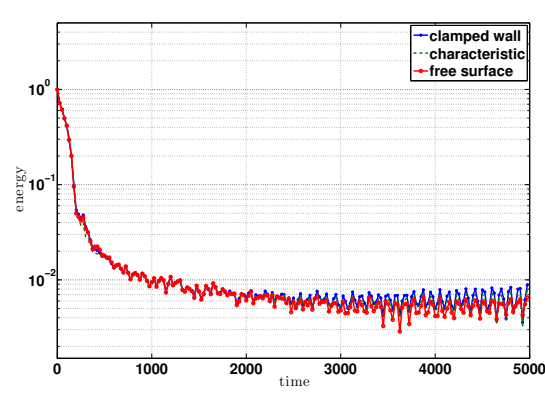

(a) Penalize all equations.

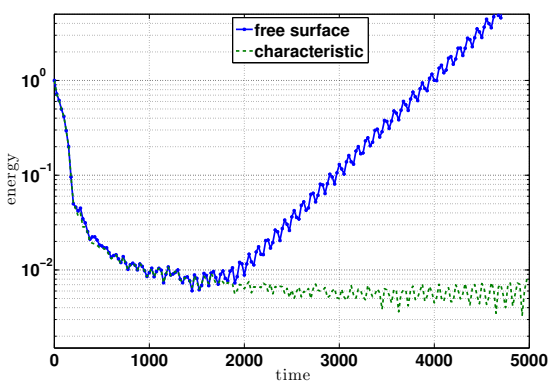

(b) Penalize velocity equations only.

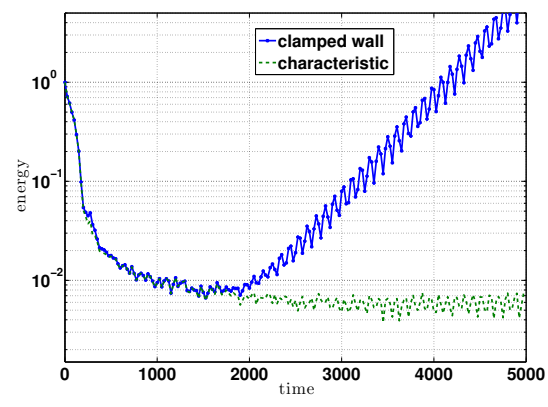

(c) Penalize stress equations only.

Figure 4: Time series of the $L_{2}$ norm of the velocity vector for the PML with the PML stabilizing penalty, $\theta_{x}=1$.

both media the S-wave speed is $c_{s}=1$. The P-wave velocity is $c_{p}=5$ in the media with $\gamma=0.2$ and $c_{p}=2.5$ in the media with $\gamma=0.4$. In figure 5 , it is apparent that the $\mathrm{P}$-wave propagates twice as fast in the medium with $\gamma=0.2$ than in the medium with $\gamma=0.4$. The $\mathrm{S}$-waves propagate at the same speed in both media. In figure $6(\mathrm{a})$ we have plotted the time history of the maximum energy on the grid. Observe that the energy decays from unity until $\sim 10^{-5}$.

\begin{tabular}{c|c|c}
$h$ & error & rate \\
\hline 1 & $1.3000 \times 10^{-3}$ & - \\
0.5 & $4.0100 \times 10^{-5}$ & 4.9817 \\
0.25 & $1.1548 \times 10^{-6}$ & 5.1179 \\
0.125 & $2.4567 \times 10^{-8}$ & 5.5548 \\
\hline
\end{tabular}

Table 1: A vertical strip PML problem: PML errors for an aluminum solid with $\gamma=0.4593$.

Next, we evaluate the accuracy of the PML. The errors introduced when a PML is used in computations can be divided into two different categories: numerical reflections and the modeling error. Numerical reflections are discrete 

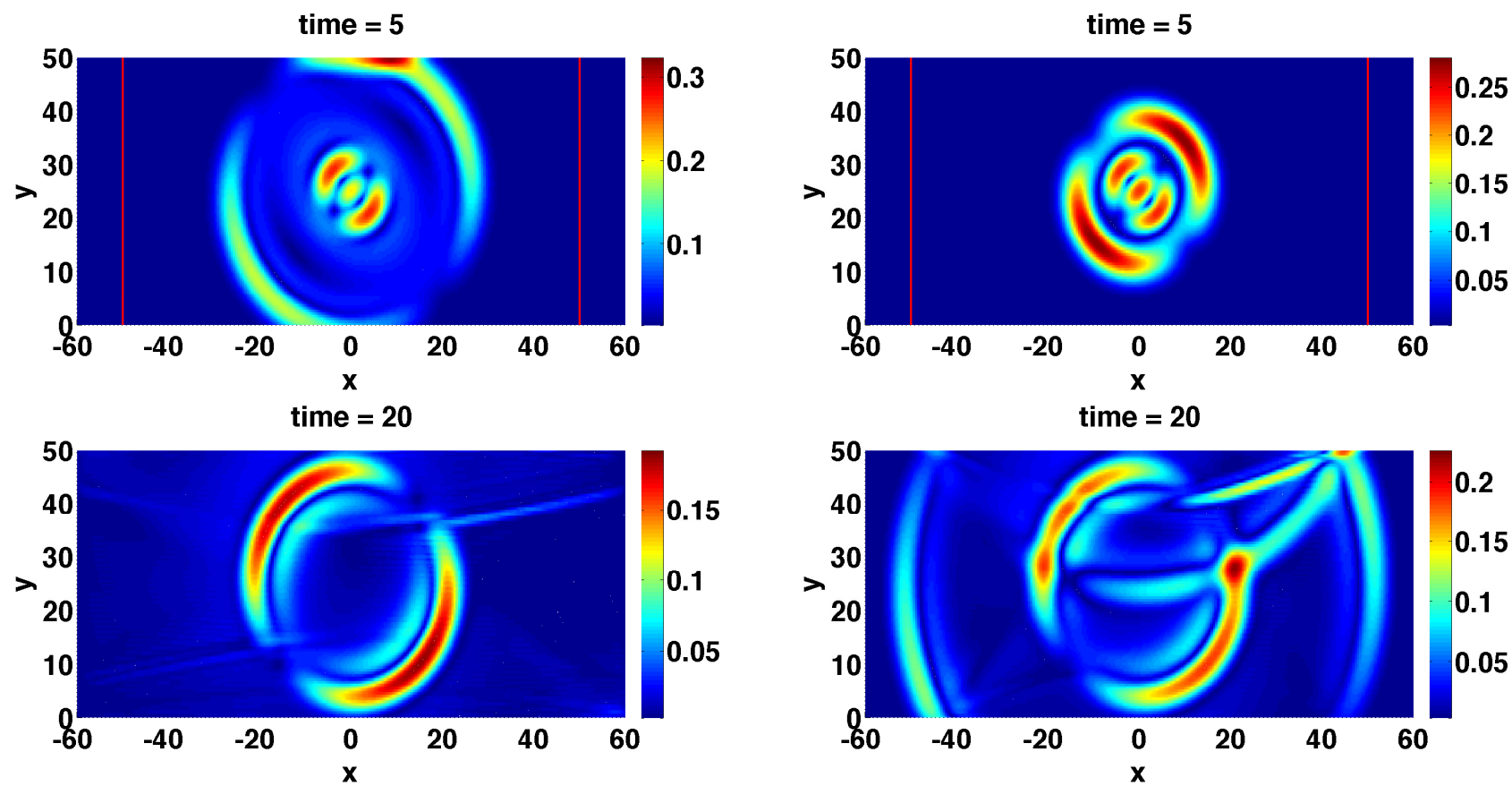

time $=\mathbf{4 0}$
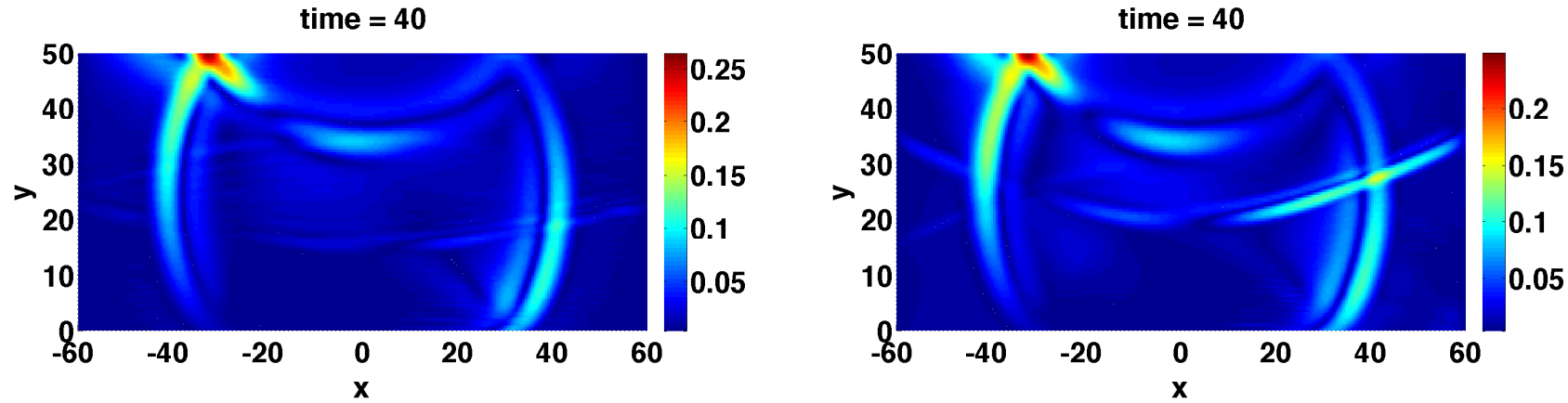

Figure 5: A vertical strip PML problem. Left column: the snapshots of the pointwise energy $\sqrt{\left(v_{x}^{2}+v_{y}^{2}\right) / 2}$ for $\gamma=0.2 ;$ Right column: the snapshots of the pointwise energy $\sqrt{\left(v_{x}^{2}+v_{y}^{2}\right) / 2}$ for $\gamma=0.4$.

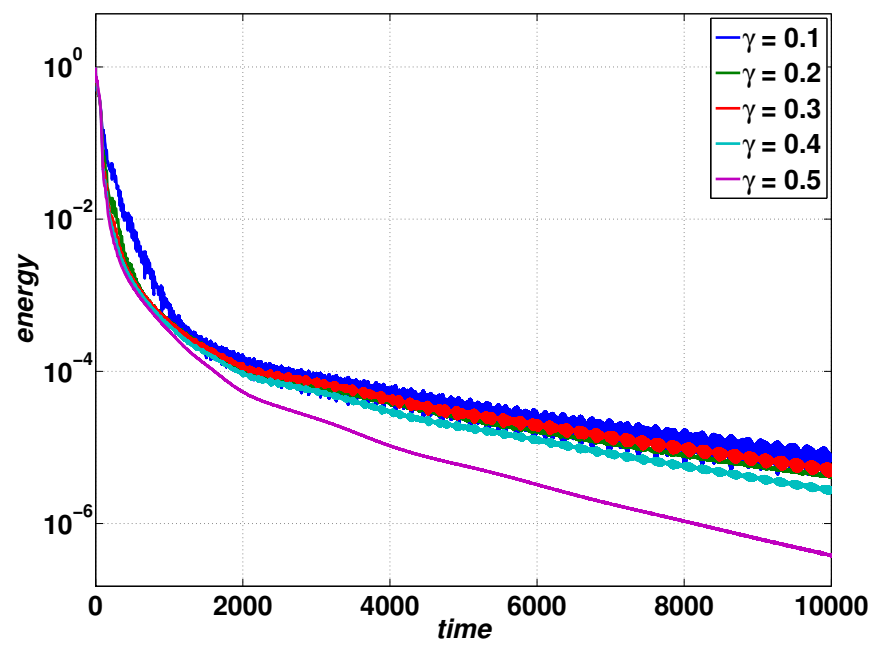

(a) Time series of the pointwise maximum energy.

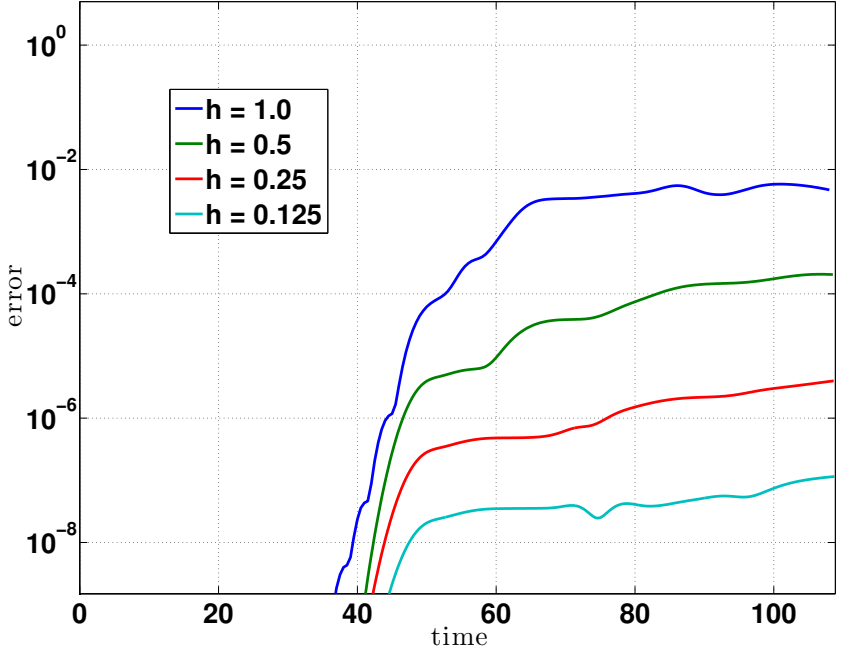

(b) Time series of PML errors for an aluminum solid.

Figure 6: A vertical strip PML problem. 
effects introduced by discretizing the PML and seen inside the computational domain. The modeling error is introduced because the layer and the magnitude of damping coefficient are finite. Numerical reflections should converge to zero as the mesh is refined. Note that the modeling error is not caused by numerical approximations, and thus is independent of the numerical method used. The modeling error is expected to decrease as the PML width or the magnitude of damping coefficient increases. For sufficiently small mesh sizes numerical reflections are infinitesimally small and the modeling error dominates the total PML error.

As before, we consider an aluminum solid defined by $\gamma=0.4593$. The corresponding $\mathrm{P}$-wave and S-wave velocities are given by $c_{p}=2.1772, c_{s}=1$. To quantify numerical errors we use the PML width, $\delta=10$ and set tol $=10^{-4} h^{4}$ so that the PML error converges at least at the rate of the interior discretization. The factor $10^{-4}$ is empirically determined. We compute the solution until $t=50 c_{p}$ so that we can account for numerical reflections and the modeling error arriving from the outer edges of the computational domain. We also compute a reference solution in a larger domain without the PML. The error is defined as the pointwise maximum difference of the reference solution and the PML solution in the interior, $(x, y) \in[-50,50] \times[0,50]$. In figure $6(\mathrm{~b})$, we have plotted the numerical error against time for various resolutions. Table 1 gives the error at the final time. Observe that the error is decreasing, and it converges to zero at a high rate, as we refine the mesh, see table 1 and figure $6(\mathrm{~b})$.

\subsection{The half-space problem with PML corners}

We consider an isotropic linear elastic half-space with the free-surface boundary condition. To perform numerical simulations we truncate the computational domain and surround the artificial boundaries with PML. The setup involves a vertical PML layer closing the left and right edges, and horizontal PML layer closing the bottom edge of the elastic block. There are also corner regions where the horizontal and vertical layers are both active. Numerical experiments in this section demonstrates that the PML with appropriate numerical boundary procedures ensure robust treatment of corners. To do this we will repeat some of the numerical experiments of last subsection, investigating stability and accuracy.

The simulation setup and parameters are similar as before, with the PML stabilizing SAT terms $\theta_{x}=\theta_{y}=1$. The only difference is that we have added a horizontal PML layer at the bottom of the elastic block, having $-\delta \leq y \leq 0$, with $\delta=10$. The damping functions $d_{x}(x), d_{y}(y)$ are a third degree monomial defined by (72). We consider the velocity ratios $\gamma=0.1,0.2,0.3,0.4,0.5$ and propagate the solution until $t=10000$. The snapshots of the solution at $t=5,20,40$ are shown in figure 7 for $\gamma=0.2,0.4$. Figure 7 shows how the initial pulse spreads, being reflected by the free-surface and the absorption of waves by the PML. Note also that all downward propagating waves are perfectly absorbed by the PML at the bottom edge of the elastic block. In figure 8(a) we have plotted the time history of the maximum energy on the grid. Observe that the energy decays from unity until $\sim 10^{-6}$.

Next we quantify numerical accuracy. As before, we consider an aluminum solid defined by $\gamma=0.4593$. To quantify numerical errors we use the PML width, $\delta=10$ and set tol $=10^{-4} h^{4}$ so that the PML error converges at least at the rate of the interior discretization. We compute the solution until $t=50 c_{p}$ so that we can account for numerical reflections and the modeling error arriving from the outer edges of the computational domain. We also compute a reference solution in a larger domain without the PML. The error is defined as the pointwise maximum difference of the reference solution and the PML solution in the interior, $(x, y) \in[-50,50] \times[0,50]$. In figure $8(\mathrm{~b})$, we have plotted the numerical error against time for various resolutions. Table 2 gives the error at the final time. Note that because the horizontal PML layer is closer to the source, elastic waves penetrate the horizontal PML layer much earlier than the vertical PML layer. From figure 8(b) it is not therefore surprising that PML errors here appear much earlier and level up as time progresses. Table 2 and figure 8(b) demonstrate that the PML error is decreasing, and it converges to zero at a high rate, as we refine the mesh. The results here is also true when the PML completely surrounds the computational domain.

\begin{tabular}{c|c|c}
$h$ & error & rate \\
\hline 1 & $5.7000 \times 10^{-3}$ & - \\
0.5 & $1.2569 \times 10^{-4}$ & 5.4963 \\
0.25 & $3.9609 \times 10^{-6}$ & 4.9878 \\
0.125 & $1.1326 \times 10^{-7}$ & 5.1281 \\
\hline
\end{tabular}

Table 2: A half-space problem with PML corners. PML errors for an aluminum solid with $\gamma=0.4593$.

\section{Concluding remarks}

We have presented an investigation of the well-posedness and stability of boundary conditions terminating the PML for the elastic wave equation in velocity-stress formulation. In the theoretical part of the paper we consider a 2D, 

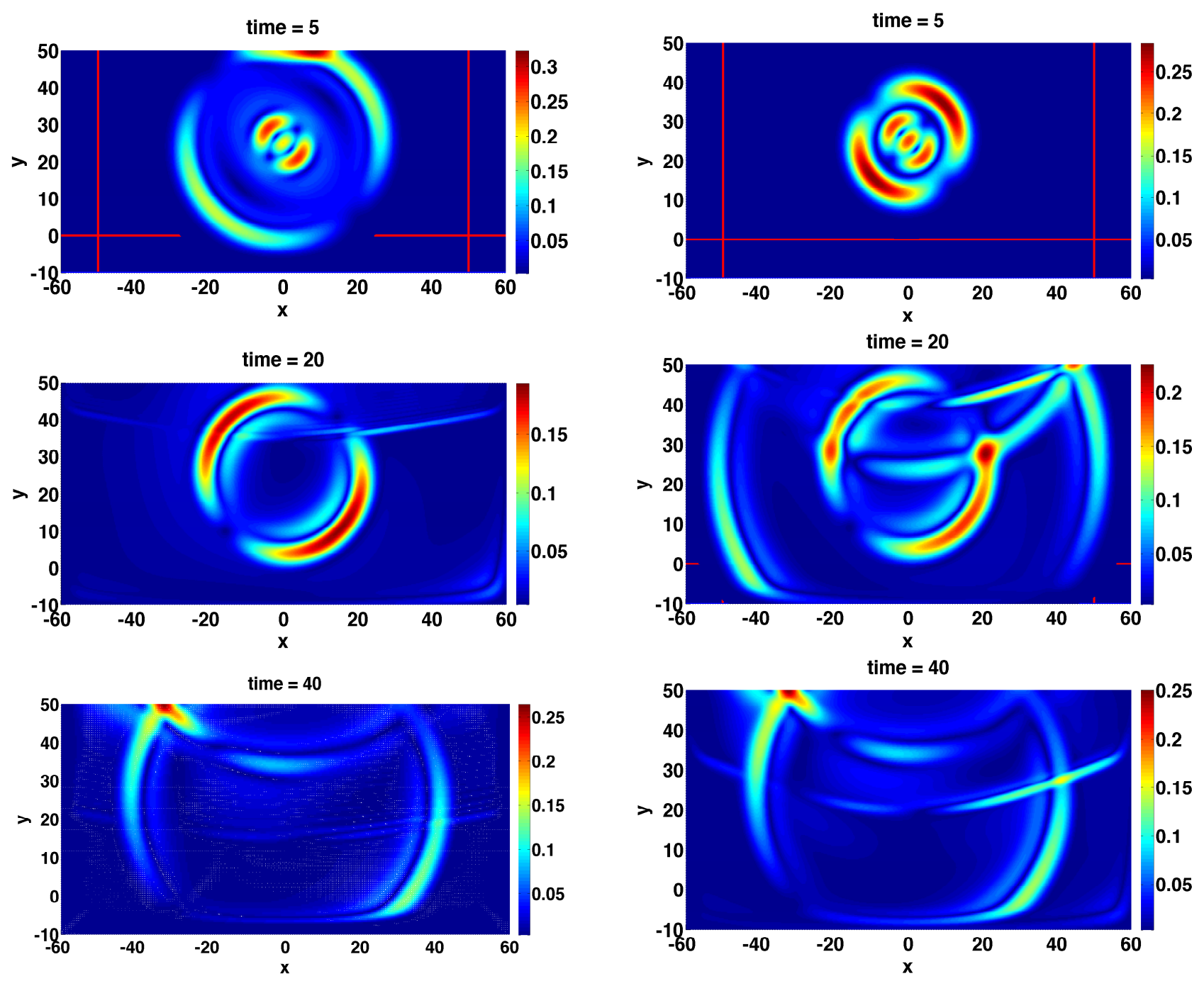

Figure 7: A half-space problem with PML corners. Left column: the snapshots of the pointwise energy $\sqrt{\left(v_{x}^{2}+v_{y}^{2}\right) / 2}$ for $\gamma=0.2 ;$ Right column: the snapshots of the pointwise energy $\sqrt{\left(v_{x}^{2}+v_{y}^{2}\right) / 2}$ for $\gamma=0.4$.

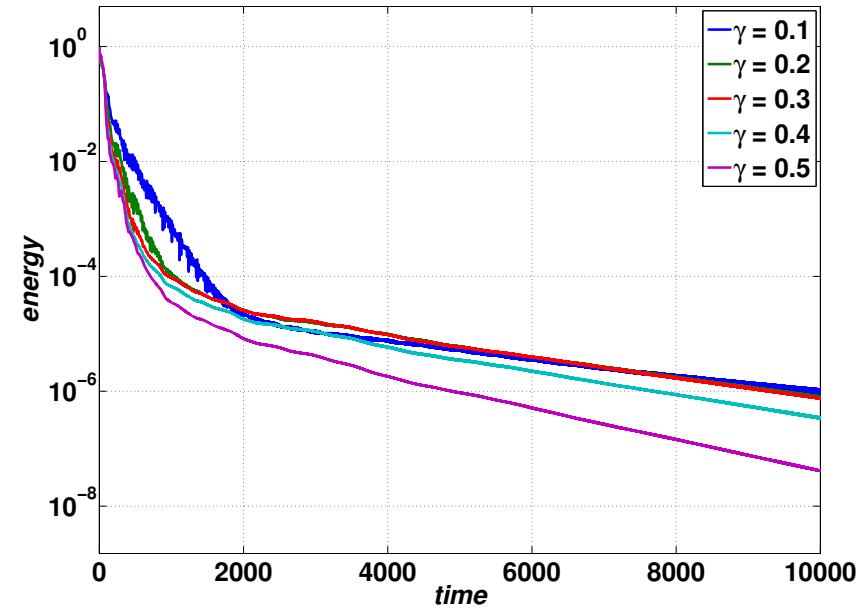

(a) Time series of the pointwise maximum energy.

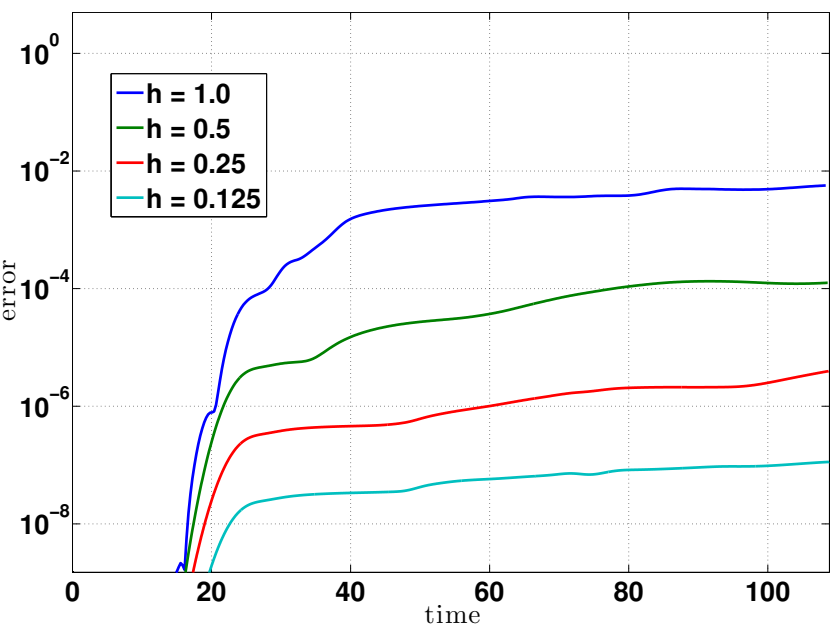

(b) Time series of PML errors for an aluminum solid.

Figure 8: A half-space problem with PML corners. 
constant coefficient PML with damping in the horizontal direction. Modal analysis in $[2,3,4,10]$ of the corresponding Cauchy problem and of half-plane problems with horizontal physical boundaries show that no growth is expected in these settings. In this paper we analyze the remaining half-plane problem, with boundary normal to the damping direction, which corresponds to the boundary closure at the outer edge of a PML. We prove that linear well-posed boundary conditions for the elastic wave equation never support growing modes when they are used as PML boundary closure.

The challenge lies in constructing accurate and stable numerical approximations for the PML and the boundary conditions. To do this, we construct a continuous energy estimate in the Laplace space for a simplified 1D problem and a 2D PML corner problem. By mimicking the continous energy estimates in the discrete setting, we develop a numerical boundary procedure using the SBP-SAT methodology, and involving special stabilizing PML penalty terms in the auxiliary equations. A classification into dissipative and non-dissipative boundary treatment is also developed. Numerical experiments for $2 \mathrm{D}$ problems with realistic variable coefficient PMLs show that the stabilizing PML penalty terms are essential for numerical stability. In addition, we find that to avoid unphysical growth the boundary procedure must be dissipative. Thus, we obtain a highly accurate and reliable domain truncation scheme.

We also note that the PML and our schemes have been successfully implemented in a large 2D finite difference open source code: FDMAP (http://pangea.stanford.edu/edunham/codes/codes.html), for wave propagation and dynamic earthquake rupture simulations. Since it can be shown that finite/spectral element schemes satisfy the SBP property $[18,29]$, we believe that the numerical boundary procedure can be extended to these methods. However, this is worth exploring further.

\section{Acknowledgements}

The work of the first author (K.D.) was supported by King Abdullah University of Science and Technology (KAUST) through a joint KAUST Academic Excellence Alliance (AEA) grant with Stanford. The second author (J.E.K.) acknowledges partial support from the National Science Foundation (OCI-1122734) and the Southern California Earthquake Center (SCEC). SCEC is funded by NSF Cooperative Agreement EAR-1033462 and USGS Cooperative Agreement G12AC20038. The SCEC contribution number for this paper is 2070.

\section{Appendix A. Some useful lemmata}

Introduce the complex number $z=x+i y$ and define the branch cut of $\sqrt{z}$ by

$$
-\pi<\arg (x+i y) \leq \pi, \quad \arg \sqrt{x+i y}=\frac{1}{2} \arg (x+i y) .
$$

The following Lemma was adapted from Lemma 6 in [16].

Lemma 1. Let $k_{x}$ be a real number and let $s=a+i b$ be a complex number where $a>0$. Consider the relation

$$
\kappa=\sqrt{s^{2}+k_{x}^{2}} .
$$

There are positive real numbers $\beta_{0} \geq 1,0<\epsilon_{0} \leq 1$ such that $\Re \kappa=\beta_{0} a$, ऽ $\kappa=\epsilon_{0} b$.

As a preliminary we introduce $d \geq 0, \alpha \geq 0$,

$$
\begin{gathered}
a^{\prime}=\frac{a}{\sqrt{(a+\alpha)^{2}+b^{2}}}, \quad b^{\prime}=\frac{b}{\sqrt{(a+\alpha)^{2}+b^{2}}}, \quad \alpha^{\prime}=\frac{\alpha}{\sqrt{(a+\alpha)^{2}+b^{2}}}, \quad d^{\prime}=\frac{d}{\sqrt{(a+\alpha)^{2}+b^{2}}}, \\
S_{x}=\frac{s+\alpha+d}{s+\alpha}=1+d^{\prime}\left(a^{\prime}+\alpha^{\prime}\right)-i d^{\prime} b^{\prime}, \quad s S_{x}=a\left(1+d^{\prime}\left(a^{\prime}+\alpha^{\prime}\right)\right)+d^{\prime} b^{\prime} b+i b\left(1+\alpha^{\prime} d^{\prime}\right) .
\end{gathered}
$$

Note that

$$
\Re\left(s S_{x}\right)=\sqrt{(a+\alpha)^{2}+b^{2}}\left(a^{\prime}\left(1+d^{\prime}\left(a^{\prime}+\alpha^{\prime}\right)\right)+d^{\prime} b^{\prime 2}\right)>0 .
$$

Lemma 2. Let $k_{x}$ be a real number and let $s=a+i b$ be a complex number where $a>0$. Consider the relations

$$
\kappa=\sqrt{s^{2}+k_{x}^{2}},
$$

There are positive real numbers $\beta_{0} \geq 1,0<\epsilon_{0} \leq 1$ such that

$$
\Re\left(S_{x} \kappa\right)=\left(\sqrt{(a+\alpha)^{2}+b^{2}}\right)\left(\beta_{0} a^{\prime}\left(1+d^{\prime}\left(a^{\prime}+\alpha^{\prime}\right)\right)+\epsilon_{0} d^{\prime} b^{\prime 2}\right)>0,
$$


Proof:

Consider $\kappa=\sqrt{s^{2}+k_{x}^{2}}=\left(\beta_{0} a+i \epsilon_{0} b\right)$ and $S_{x}=1+d^{\prime}\left(a^{\prime}+\alpha^{\prime}\right)-i \sigma^{\prime} b^{\prime}$, then we have

$$
\begin{aligned}
& S_{x} \kappa=\left(1+d^{\prime}\left(a^{\prime}+\alpha^{\prime}\right)-i d^{\prime} b^{\prime}\right)\left(\beta_{0} a+i \epsilon_{0} b\right), \\
& \Longrightarrow \Re\left(S_{x} \kappa\right)=\left(\sqrt{(a+\alpha)^{2}+b^{2}}\right)\left(\beta_{0} a^{\prime}\left(1+d^{\prime}\left(a^{\prime}+\alpha^{\prime}\right)\right)+\epsilon_{0} d^{\prime} b^{2}\right)>0 .
\end{aligned}
$$

As a consequence of lemmas 1 and 2 we have the following:

Corollary 1. Let $\gamma>0$ and $k_{y}$ be real numbers, and let $s=a+i b$ be a complex number where $a>0$. Consider the relations

$$
\kappa_{1}=\frac{1}{\gamma} \sqrt{\left(s S_{x}\right)^{2}+\left(\gamma S_{x} k_{y}\right)^{2}}, \quad \kappa_{2}=\sqrt{\left(s S_{x}\right)^{2}+\left(S_{x} k_{y}\right)^{2}} .
$$

For all real numbers $\sigma, \alpha \geq 0$, the complex numbers $\kappa_{1}, \kappa_{2}$ have positive real parts $\Re \kappa_{1}>0, \Re \kappa_{2}>0$.

\section{Appendix B. Proof of theorem 2}

\section{Appendix B.1. Clamped wall boundary condition}

Consider first the case of a clamped boundary with $r_{x}^{(x)}=r_{y}^{(x)}=-1$, implying $\mathbf{I}+\mathbf{R}=0$. Thus, from (30) we have

$$
\left(\theta_{1} \boldsymbol{\Phi}_{1}+\theta_{2} \boldsymbol{\Phi}_{2}\right)=0 \Longleftrightarrow\left(\boldsymbol{\Phi}_{1}, \boldsymbol{\Phi}_{2}\right)\left(\begin{array}{c}
\theta_{1} \\
\theta_{2}
\end{array}\right)=0
$$

Since the eigenvectors are complete we must have

$$
\mathcal{C}\left(s, k_{y}, r_{x}^{(x)}, r_{y}^{(x)}\right):=\operatorname{det}\left(\boldsymbol{\Phi}_{1}, \boldsymbol{\Phi}_{2}\right) \neq 0, \quad \forall k_{y} \in \mathbb{R}, \quad \Re s>0,
$$

implying $\theta_{1}=\theta_{2}=0$, for all $\Re s>0$. Thus the clamped boundary condition (32a) does not support boundary wave modes.

\section{Appendix B.2. Free surface boundary condition}

Consider now the free surface boundary condition with $r_{x}^{(x)}=r_{y}^{(x)}=1$, implying $\mathbf{I}-\mathbf{R}=0$. From (30) we have

$$
\frac{1}{s}\left(\begin{array}{cc}
\left(k_{y}+\frac{\kappa_{1}^{2}}{k_{y}}\right) \gamma^{2} i & 2 k_{y} \gamma^{2} i \\
2 \kappa_{1} \gamma^{2} & \kappa_{2}+\frac{k_{y}^{2}\left(2 \gamma^{2}-1\right)}{\kappa_{2}}
\end{array}\right)\left(\begin{array}{c}
\theta_{1} \\
\theta_{2}
\end{array}\right)=0,
$$

thus yielding the determinant condition

$$
\frac{1}{s^{2}} \mathcal{C}_{0}\left(s, k_{y}\right) \equiv \operatorname{det}\left(\frac{1}{s}\left(\begin{array}{cc}
\left(k_{y}+\frac{\kappa_{1}^{2}}{k_{y}}\right) \gamma^{2} i & 2 k_{y} \gamma^{2} i \\
2 \kappa_{1} \gamma^{2} & \kappa_{2}+\frac{k_{y}^{2}\left(2 \gamma^{2}-1\right)}{\kappa_{2}}
\end{array}\right)\right) \neq 0, \quad \Re s>0 .
$$

Here, $\mathcal{C}_{0}\left(s, k_{y}\right)$ is the Rayleigh dispersion relation defined by

$$
\mathcal{C}_{0}\left(s, k_{y}\right) \equiv\left(s^{2}+2 \gamma^{2} k_{y}^{2}\right)^{2}-4 \gamma^{4} k_{y}^{2} \sqrt{s^{2}+k_{y}^{2}} \sqrt{\frac{s^{2}+\gamma^{2} k_{y}^{2}}{\gamma^{2}}}=0 .
$$

The equation $\left(1 / s^{2}\right) \mathcal{C}_{0}\left(s, k_{y}\right)=0$ has exactly two non zero, purely imaginary solutions $s=i \beta, \beta \in \mathbb{R}$ for all $0<\gamma^{2}<1$ and $k_{y} \neq 0$.

The proof for $s=i \beta$, can be easily adapted from [16]. Consider

$$
\lim _{s \rightarrow 0}\left(1 / s^{2}\right) \mathcal{C}_{0}\left(s, k_{y}\right)=\lim _{s \rightarrow 0} \frac{\frac{\partial \mathcal{C}_{0}\left(s, k_{y}\right)}{\partial s}}{\frac{\partial\left(s^{2}\right)}{\partial s}}=2 \gamma^{2}\left(1-\gamma^{2}\right) k_{y}^{2} \neq 0 .
$$

Therefore, $s=0$ is not a solution of $\left(1 / s^{2}\right) \mathcal{C}_{0}\left(s, k_{y}\right)=0$. The purely imaginary roots are called generalized eigenvalues and correspond to Rayleigh wave modes propagating on the surface of an elastic solid without decay. This is in contrast to the free surface boundary conditions at $y=0$, where all Rayleigh wave modes are damped by the PML, see $[10,4]$. 
Appendix B.3. Characteristic boundary condition

Finally, consider the characteristic boundary condition with $r_{x}^{(x)}=r_{y}^{(x)}=0$, implying $\mathbf{R}=0$. From (30) we have

$$
\frac{1}{s}\left(\begin{array}{cc}
-k_{y}\left(\frac{s}{\kappa_{1}}+2 \gamma^{2}\right) i & -\frac{\left(\kappa_{2}^{2}+s \kappa_{2}+\left(2 \gamma^{2}-1\right) k_{y}^{2}\right) i}{k_{y}} \\
\frac{\kappa_{1}^{2} \gamma^{2}+s \kappa_{1} \gamma+k_{y}^{2} \gamma^{2}}{\kappa_{1}} & 2 \kappa_{2} \gamma^{2}+s \gamma
\end{array}\right)\left(\begin{array}{c}
\theta_{1} \\
\theta_{2}
\end{array}\right)=0
$$

yielding the determinant condition

$$
\frac{1}{s^{2}} \mathcal{C}_{0}\left(s, k_{y}\right) \equiv \operatorname{det}\left(\frac{1}{s}\left(\begin{array}{cc}
-k_{y}\left(\frac{s}{\kappa_{1}}+2 \gamma^{2}\right) i & -\frac{\left(\kappa_{2}^{2}+s \kappa_{2}+\left(2 \gamma^{2}-1\right) k_{y}^{2}\right) i}{k_{y}} \\
\frac{\kappa_{1}^{2} \gamma^{2}+s \kappa_{1} \gamma+k_{y}^{2} \gamma^{2}}{\kappa_{1}} & 2 \kappa_{2} \gamma^{2}+s \gamma
\end{array}\right)\right) \neq 0, \quad \Re s>0 .
$$

The function $\mathcal{C}_{0}\left(s, k_{y}\right)$ is define by

$$
\begin{aligned}
\mathcal{C}_{0}\left(s, k_{y}\right) & =\frac{\gamma}{k_{y} \sqrt{s^{2}+\gamma^{2} k_{y}^{2}}} \times \\
& \left(\left(s^{2}+2 \gamma^{2} k_{y}^{2}\right)^{2}+2 \gamma^{2} k_{y}^{4}-\gamma s^{2} k_{y}^{2}+\left(\sqrt{s^{2}+\gamma^{2} k_{y}^{2}}+\sqrt{s^{2}+k_{y}^{2}}\right) s^{3}+\sqrt{s^{2}+\gamma^{2} k_{y}^{2}} \sqrt{s^{2}+k_{y}^{2}}\left(s^{2}-4 \gamma^{3} k_{y}^{2}\right)\right) .
\end{aligned}
$$

We will now characterize the solutions of the determinant the equation $\mathcal{C}_{0}\left(s, k_{y}\right)=0$.

We know that the equation $\mathcal{C}_{0}\left(s, k_{y}\right)=0$, has no root $s$, with $\Re s>0$. If $\Re s>0$ then there is energy growth $\mathrm{E}_{\gamma}(t)>\mathrm{E}_{\gamma}(0)$, which is in contradiction with (11), and thus $\Re s \leq 0$.

To investigate generalized eigenvalues, that is the limit $\Re s \rightarrow 0$, we introduce $\bar{s}^{2}=s^{2} / k_{y}^{2}$ and consider the equation

$$
\mathcal{C}_{1}(\bar{s}) \equiv\left(\bar{s}^{2}+2 \gamma^{2}\right)^{2}+2 \gamma^{2}-\gamma \bar{s}^{2}+\left(\sqrt{\bar{s}^{2}+\gamma^{2}}+\sqrt{\bar{s}^{2}+1}\right) \bar{s}^{3}+\sqrt{\bar{s}^{2}+\gamma^{2}} \sqrt{\bar{s}^{2}+1}\left(\bar{s}^{2}-4 \gamma^{3}\right)=0 .
$$

Note that

$$
\frac{1}{s^{2}} \mathcal{C}_{0}\left(s, k_{y}\right) \equiv \frac{\gamma}{\bar{s}^{2} \sqrt{\bar{s}^{2}+\gamma^{2}}} \mathcal{C}_{1}(\bar{s})=0 .
$$

We will show that the equation (B.6) has no solution $\bar{s}=0$ or $\bar{s}=i \xi, \xi \in \mathbb{R}$ for all $0<\gamma^{2}<1$. Inserting $\bar{s}=0$ in (B.6) we have $\mathcal{C}_{1}(0)=2 \gamma^{2} \neq 0$ for all $0<\gamma^{2}<1$, and thus $\bar{s}=0$ is not a solution.

Now if $\bar{s}=i \xi$ is a solution, then (B.6) becomes

$$
\left(2 \gamma^{2}-\xi^{2}\right)^{2}+2 \gamma^{2}+\gamma \xi^{2}-i\left(\sqrt{\left(\gamma^{2}-\xi^{2}\right) \xi^{2}}+\sqrt{\left(1-\xi^{2}\right) \xi^{2}}\right) \xi^{2}-\sqrt{\gamma^{2}-\xi^{2}} \sqrt{1-\xi^{2}}\left(4 \gamma^{3}+\xi^{2}\right)=0 .
$$

Considering first $0<\xi^{2}<\gamma^{2}$ and collecting the real and imaginary parts separately yields two equations

$$
\begin{gathered}
\left(\sqrt{\left(\gamma^{2}-\xi^{2}\right) \xi^{2}}+\sqrt{\left(1-\xi^{2}\right) \xi^{2}}\right) \xi^{2}=0, \\
\left(2 \gamma^{2}-\xi^{2}\right)^{2}+2 \gamma^{2}+\gamma \xi^{2}-\sqrt{\gamma^{2}-\xi^{2}} \sqrt{1-\xi^{2}}\left(4 \gamma^{3}+\xi^{2}\right)=0,
\end{gathered}
$$

which must both be satisfied simultaneously. From equation (B.9), the only possible solution is $\xi=0$, which is a contradiction. Now consider $\xi^{2}=\gamma^{2}$ and insert in (B.8) above we have

$$
\gamma^{4}+2 \gamma^{2}+\gamma^{3}-i \sqrt{\left(1-\gamma^{2}\right) \gamma^{2}} \gamma^{2}=0 .
$$

Collecting the real and the imaginary parts separately gives

$$
\gamma^{4}+2 \gamma^{2}+\gamma^{3}=0, \quad \sqrt{\left(1-\gamma^{2}\right) \gamma^{2}} \gamma^{2}=0
$$

Since $0<\gamma^{2}<1$, these are contradictions. There are no solutions of (B.8) for $0<\xi^{2} \leq \gamma^{2}$.

Now turning to $\gamma^{2}<\xi^{2}<1$ gives, upon collecting real and imaginary terms, the two conditions

$$
\begin{gathered}
\left(2 \gamma^{2}-\xi^{2}\right)^{2}+2 \gamma^{2}+\gamma \xi^{2}-i \sqrt{\left(\gamma^{2}-\xi^{2}\right) \xi^{2}} \xi^{2}=0, \\
i \sqrt{\left(1-\xi^{2}\right) \xi^{2}} \xi^{2}-\sqrt{\gamma^{2}-\xi^{2}} \sqrt{1-\xi^{2}}\left(4 \gamma^{3}+\xi^{2}\right)=0 .
\end{gathered}
$$

Since the square root in (B.13) is purely imaginary, (B.13) cannot vanish yielding a contradiction. On the other hand if $\xi^{2}=1$ then (B.8) becomes

$$
\left(2 \gamma^{2}-1\right)^{2}+2 \gamma^{2}+\gamma-i \sqrt{\left(\gamma^{2}-1\right)}=0 .
$$

This again yields a contradiction, as the square root is purely imaginary and there are no solutions of (B.8) for $\gamma^{2}<\xi^{2} \leq 1$

The only remaining case is $\xi^{2}>1$. In this case all the square roots in (B.8) are purely imaginary and every term is positive and nonzero. Thus there are no solutions $\xi^{2}>1$ satisfying (B.8).

Thus all solutions of the equation $1 / s^{2} \mathcal{C}_{0}\left(s, k_{y}\right)=0$, defined in (B.4), must satisfy $\Re s<0$ for all $\sigma, \alpha \geq 0$. There are neither zero roots nor purely imaginary roots. 


\section{References}

[1] J-P. Bérenger, A perfectly matched layer for the absorption of electromagnetic waves, J. Comput. Phys., 114, 185-200, (1994).

[2] E. Bécache, S. Fauqueux, P. Joly, Stability of perfectly matched layers, group velocities and anisotropic waves, J. Comput. Phys., 188, 399-433, (2003).

[3] D. Appelö and G. Kreiss, A new absorbing layer for elastic waves, J. Comput. Phys. 215, 642-660, (2006).

[4] K. Duru and G. Kreiss, Boundary waves and stability of the perfectly matched layer for the two space dimensional elastic wave equation in second order form, SIAM Num. Analys., Vol. 52, No. 6, pp. 2883-2904, (2014).

[5] E. A. Skelton, S.D.M. Adams and R. V. Craster, Guided elastic waves and perfectly matched layers, Wave Motion, $44,573-592,(2007)$.

[6] C. Zeng, J. Xia, R. Miller and G. Tsoflias, Application of the multi-axial perfectly matched layer (M-PML) to near-surface seismic modeling with Rayleigh waves, Geophysics 76(3), 43-52, (2011).

[7] M. Kuzuoglu and R. Mittra, Frequency Dependence of the Constitutive Parameters of Causal Perfectly Matched Anisotropic Absorbers, IEEE Microw. and Guided Wave Lett., 6, (1996).

[8] Z. Xie, D. Komatitsch, R. Martin and R. Matzen, Improved forward wave propagation and adjoint-based sensitivity kernel calculations using a numerically stable finite-element PML, Geophys. J. Int. 198 (3): 1714-1747, (2014).

[9] K. Duru, G. Kreiss, A Well-posed and discretely stable perfectly matched layer for elastic wave equations in second order formulation, Commun. Comput. Phys., 11, 1643-1672, (2012).

[10] K. Duru, Perfectly matched layers and high order difference methods for wave equations, PhD Thesis Uppsala University, (2012).

[11] K. Duru, The role of numerical boundary procedures in the stability of perfectly matched layers, submitted to SIAM Sc. Comput., (2014).

[12] B. Sjögreen, N. A. Petersson, Perfectly matched layer for Maxwell's equation in second order formulation, J. Comput. Phys., 209, 19-46, (2005).

[13] L. Halpern, S. Petit-Bergez, J. Rauch, The analysis of matched layers, Conflu. Math. 3(2), 159-236 (2011).

[14] D. Appelö and T. Colonius, A high order super-grid-scale absorbing layer and its application to linear hyperbolic systems, J. Comput. Phys., 228 (11), 4200-4217, (2009).

[15] J. Tago, L. Métivier and J. Virieux, SMART layers: a simple and robust alternative to PML approaches for elastodynamics, Geophys. J. Int. (2014) 199 (2): 700-706.

[16] H.-O. Kreiss, and N.A. Petersson, Boundary estimates for the elastic wave equations in almost incompressible materials, SIAM J. Num. Anal. 50, 1556-1580, (2012).

[17] H.-O. Kreiss, J. Lorenz, Initial-Boundary Value Problems and the Navier-Stokes Equations, Pure and Appl. Math., vol 136. Academic Press, Boston, USA, 1989.

[18] H.-O. Kreiss, and G. Scherer, Finite element and finite difference methods for hyperbolic partial differential equations, Mathematical aspects of finite elements in partial differential equations, Academic Press, New York, (1974).

[19] J. D. Achenbach, Wave propagation in elastic solids, vol. 16 of Applied Mathematics and Mechanics. NorthHolland, (1973).

[20] J. Kozdon, E. Dunham, J. Nordström, Simulation of dynamic earthquake ruptures in complex geometries using high-order finite difference methods, J Sci Comput. DOI 10.1007/s10915-012-9624-5 (2012).

[21] D. Givoli, High-order local non-reflecting boundary conditions: a review, Wave Motion 39:319-326, (2004).

[22] T. Hagstrom, T. Warburton and D. Givoli, Radiation boundary conditions for time-dependent waves based on complete plane waves expansions, J. Comput. Appl. Math. 234(16), 1988-1995 (2010). 
[23] M. H. Carpenter, D. Gottlieb, S. Abarbanel, Time-stable boundary conditions for finite-difference schemes solving hyperbolic systems: methodology and application to high-order compact schemes, J. Comput. Phys, 111(2), p 220-236 (1994).

[24] M. Svärd, On coordinate transformation for summation-by-parts operators, J. Sci. Comput., 20 (1), (2004).

[25] K. Mattsson, F. Ham, G. Iaccarino, Stable boundary treatment for the wave equation in second-order form, J Sci Comput, 41, p336-383, (2009).

[26] B. Gustafsson, High order difference methods for time dependent PDE, Springer-Verlag, Berlin Heidelberg, (2008).

[27] K. Duru and E. M. Dunham, Dynamic earthquake rupture simulations on nonplanar faults embedded in $3 D$ geometrically complex, heterogeneous elastic solids, submitted to J. Comput. Phys., (2014)

[28] B. Strand, Summation by parts for finite difference approximations for $d / d x$, J. Comput. Phys. (110) 47-67, (1994).

[29] G. J. Gassner, A skew-symmetric discontinuous Galerkin spectral element discretization and its relation to SBPSAT finite difference methods, SIAM J. Sci. Comput., 35 (2013), A1233-A1253.

[30] H.-O. Kreiss, Initial boundary value problems for hyperbolic systems, Comm. Pure Appl. Math., (23) 277-298, (1970).

[31] M. Motamed, H-O. Kreiss, Hyperbolic initial boundary value problems which are not boundary stable, Numerical Analysis, School of Computer Science and Communication, KTH, Stockholm, Sweden (2008).

[32] B. Gustafsson, The Godunov-Ryabenkii condition: The beginning of a new stability condition, Technical report 1999-014, Department of Information Technology, Uppsala University, (1999).

[33] J. Nordstrom, Error bounded schemes for time-dependent hyperbolic problems, SIAM J. Sci. Comput., 30(1), 46-59 (2007). 